\title{
Houle dans un canal présentant un passage en charge
}

\section{Wave action in a flume containing a submerged culvert}

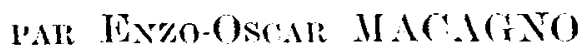

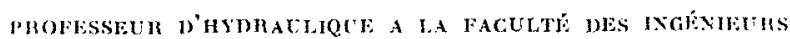

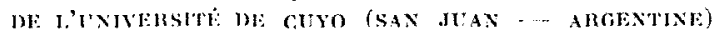

\begin{abstract}
Linstallation expérimentale: batteur, contuergeni, canal d'élude $(14 \mathrm{~m} \times 0,30 \mathrm{~m})$, filtres, amorlisseurs, appareils de mesure. - lin obslacle immergé crée un passage en charge. Les phinonienes : clapotis partiel a l'amont; houl' monrtie a l'abal; mounement oscillatoire en masse dans le passage en charge. -- Tentalive de ralcul theorique de lamortissement de la houle transmise el de la honle réfléchic par rapport à la houle incirlente; déphasages; perte d'énergie. - Résultals experimentaux; disrussion.
\end{abstract}

\begin{abstract}
Exprimental installation; ane mashine, connergent, lest runal $(1 / \mathrm{m} \times 0.30 \mathrm{~m})$, filters, dampers, measuring instruments. An immersed obstacle created a submerged culvert. The phenomenu: pertial clapotis to the npstream; damped wane to the downstream; general oscillation movement in the subnerged culvert. dllempt at theorelical calculation of damping of the transmitled and reflected wanes in relalion lo the incidental mave; difference in phases; loss of encrgy. Exporimenlal results; discussion.
\end{abstract}

\section{AVANT-PROPOS}

Ces recherehes ont été entreprises à la faveur d'un sćjour à Grenoble comme boursier du Gouvernement francais. J'exprime ici ma vive reconmaissance à M. le Ministre des Affaires Etrangères et aux Représentants diplomatiques de la France on Argentine.

Je dois aussi exprimer ma gratitude à M. Kravtchenko, Professeur à la Faculté des Sciences de Grenoble, dont les encouragements m'ont été si précieux et à qui je suis redevable d'indications et d'enseignements tres uliles, of i M. Santon, Prolesseur et Directeur du Laboraloire d'Hydraulique et d'Aérodynamique de l'Université de Grenoble, pour sa hienveillance el l'aide qu'il m'a prêtée en toute occasion.
La partie expérimentale des recherches a été effectuée au Laboratoire Dauphinois d'Hydraulique. Je remercie la Direction des Elablissements Neyrpic de la cordiale hospitalité qui m'a été ofrerte et des facilités qui mont été accordées. te dois en particulier remercier M. P. DANEs, qui m’a suggéré le sujet de Ia présente étude, et M. F. Brésfr, dont l'aide m'a été en tous points si précieuse. Mais ma gratitude ra aussi aux nembres du Laboratoire auprès desquels j’ai trouve toujours la meilleure bonne volonte.

Je veux aussi exprimer ma gratitude à Mme Macagno qui, proche ou lointaine, a trouve loujours la façon de me donner son appui ef son encouragement. 


\section{CHAPITRE I}

\section{INTRODUCTION}

Presque tous les canaux et les réseaux de camaux sont soumis à des régimes variables soit en permanence, soil transitoirement. Dans les ré seaux d'irrigation, on doit faire varier les débits pour faire une distribution de l'eau adaptée aux besoins des usagers, qui varient avec la qualité du terrain, les cultures, les saisons. Les canaux des centrales hydroélectriques ont un régime imposé par la puissance que doit fournir la centrale, qui est, en général, variable avec le temps. Les réseaux des canaux de drainage ont un régime essentiellement variable. Les niveaux dans les canaux de navigation sont soumis à des varialions par les bateaux et les éclusées. Il y a des ranaux qui sont soumis aux niveaux variables de la marée et de la houle. Le vent peut produire dans les canaux, dans certaines conditions, une houle dont l'amplitude et les effets ne sont pas négligeables.

D'autre part, l'interposition d'une conduite ou d'une galerie en charge entre deux biefs de canal, est réquente.

Le passage en charge, avec régine variable, pose donc un problème pratique, que l'on a choisi comme sujet d'étude. Parmi les différents types de mouvements variables on a choisi la houle.

Le problème est donc le suivant : la houle dans un. canal de fond horizontal séparé en deux parties par un passage en charge.

La réalisation du phénomène a été accomplie sur un canal à houle du Laboratoire Datuphinois d'Hydraulique. Dans une extrémité on a un batteur qui produit la houle et dims l'autre une plage absorbante pour crécr la condition de longueur indéfinie sur ce côté. Dans la partic centrale du canal, on place un obstacle donl l'onfoncement est variable et qui donne heu at passage en charge (fig. 1, 2, 3).

Il s'agit d'étudier la réflexion de la houle sur l'obstacle et dans le hief qu'on ajprellera damont, et la transmission de la lioule dans le bief qu'on désignera d'aval.

Les paramitres dont on itudiera l'influence seront la longueur et l'enfoneement de l'obstacle d'une part; la période el l'amplitude de la houle, d'autre part.

Ia seule itude expérimentale de notre comnaissance, présentant une analogic étroile avec la nòtre, est due à M. SkLtevam : Amortissement de la houle sous une banne plate (Rapport 2.375, non publié -- Laboratoire Dauphinois d'Hydaalique). Cette étude consistait à mesurer lamplitude de la houle pure, sous la vanne, puis l'amplitude de la houle transmise a l'aval de la vamme, pour différents enfoncements de celle-ci.

Une étude analogue a élé faite aussi par M. Chapts, avec une placpue mince, au laborattoire Dauphinois d'Hydraulique.

Une étude théorique a élé publice par M. I'nsfus. : The effect of a pertical barrier on wabes in deep water (Admirally Rescarch Laboralory, Taddington, Middlessex, 1945), qui donne le coefficient de transmission et le coefficient de reflexion. Dans cette dtude la profondeur d'eau est supposée infinie, et l'obstacle plan, d'éprisseur nulle.

\section{CHAPITRE II}

\section{INSTALLATION ET APPAREILS DE MESURE}

\section{I. - Canal à houle}

L'étude expérimentale a été réalisée dans un canal à houle du Laboratoire Dauphinois d'Hydraulique. Ce canal a été mis au point pour des ćtudes de la physique de la houle. Une description complète de l'installation a déjà été présentée par M. A. WaLlet (la Houille Blanche, $\mathrm{n}^{\circ}$ 6, 1952 , p. 843).

Avec cette installation on a pu obtenir une houle particulièrement pure et constante, comme le montrent les enregistrements photographi- ques faits au Laboratoire. Parmi les etudes réalisées sur cette installation nous pouvons $\mathfrak{i -}$ ter : l'étude des profils de la houle, des trajecloires internes de la houle et du clapolis, des courants internes de la houle, de la cólérité de la houle.

L'installation comprend successivement (fig. 1):

$$
\text { a) Partie tariali. }
$$

Elje est constituée par trois d́léments mitalliques de section rectangulaire de $75 \mathrm{~cm}$ dr lar- 
de Jinstallation d'essai.
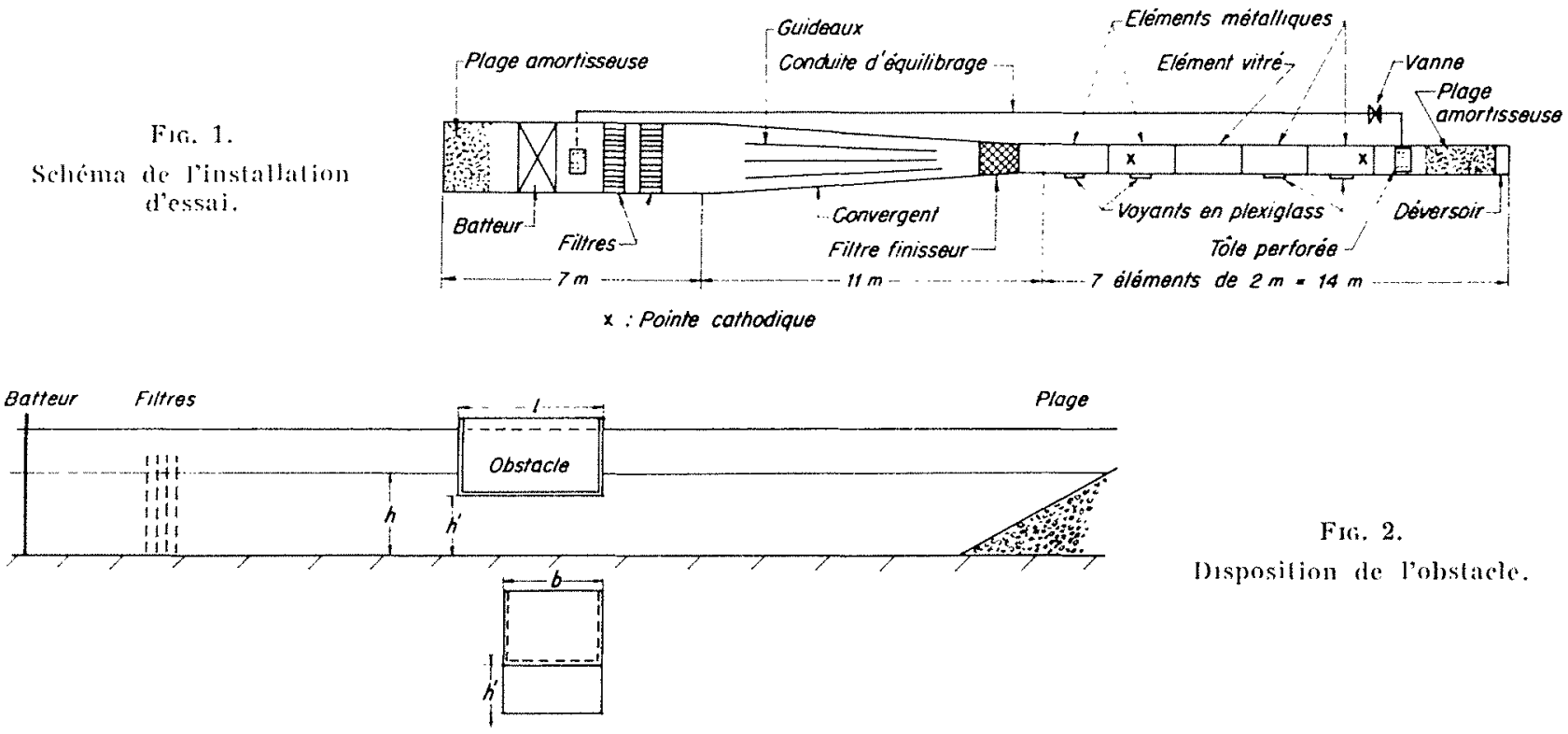

Fic. 2.

Disposilion de l'obstacle. geur el d'une longueur lotale de $7 \mathrm{~m}$. Dans celle partic se trouve le halleur qui produil la houle.

\section{b) Convergent}

Il fail le raceordement de la partie large avec le canal de section rectangulaire large de $30 \mathrm{~cm}$. La convergenee est faible pour éviter les perturbations gui auraient lieu avec une variation rapide de largeur, comme les balancements transversaux et les réflexions. La variation de la largetu de $45 \mathrm{~cm}$ se fait sur une longueur de $11 \mathrm{~m}$, el cela au moyen d'un are de parabole, un segment de droile ef un are de cubique ayant courbure nulle au point de raccordement.

Le rôle du convergent est de renforcer sans perturbations l'amplitude de la houle produite par le batteur. La convergence a été prévue de maniere à compenser la perte d'inergie dans les filtres, ainsi que l'amortissment propre.

\section{c) Canal DÉTUde.}

Il comporte sept déments mélalliques de $2 \mathrm{~m}$ de longueur. L'élément central a ses parois viIrées en vue des observations visuelles el de la photographie. Les autres éléments portent dans la partic centrale des hublots en plexiglass.

\section{d) Condutte n'loumingage.}

Cetle conduile était deslinée à ćtablir l'équilibrage dans les cas d'ouvages franchissables par la houle qui peuvent créer une accumulalion d'eau; on l'a maintenue fermée pendant notre étude.

\section{f) BatTELR A HOULE.}

Le balleur est du lype a volet plan oscillant. mais il posscde, par rapport aux batteurs classi- ques, l'avantage du réglage en hauteur de l'axe virtuel de rotation. La figure 4 montre le schéma de fonctionnement du batteur. la manivelle OM est entraînée par un moteur électriçuc
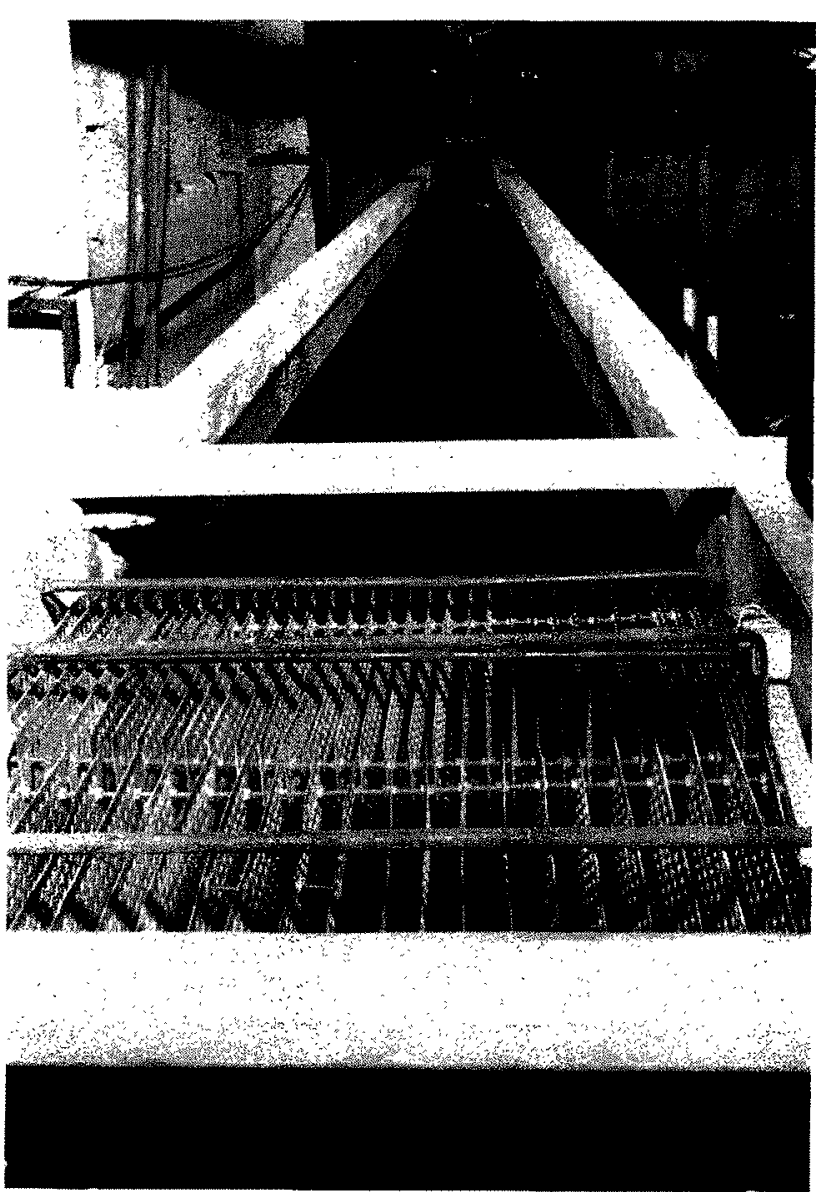

Fif. 3. - Le comvergent et les toles perforés. 
par l'intermédiaire d'un rariateur de vilesses, et elle entraine à son tour la bielle ME. L'axe A est fixe, tandis que l'axe 1) est réglable et peul occuper diverses positions $D_{1}, D_{2}, D_{: 3} \ldots$ B el $C$ sont de simples chamieres. FG represente te volet.

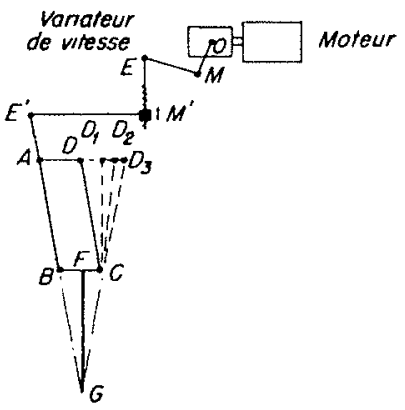

Fig. 4.

Schemat de fonctionnement du batteur.

Le centre instantane de rotation da volet est alors fonction de la position de l'axe J). S'il se trouve dans la position 1 , le volet subit un mourement de translation, qui convient a la pro- duction des houles de grande longueur dionde par rapport a ba profondeur deatu. Une position du centro de rotation qui se rapproche du fond du canal eonvient pour les houles courtes.

La periode se regile par le varialeur de vilesses. L'amplitude est réglable à distance. Pour oblenir cela, on a dispose deux bielles suceessives ME of $M^{\prime} F^{\prime}$ entre lesquelles est interposé un levier intermediaire, qui est allaque par la premicre bielle on un poinl variable grâce à un conlisseau mù par un moleur blectrique. En faisaml lourner ee moteur dans le sens voulu, lamplitude. du moturement peut etre reghle it distance ffig. $\overline{5}$, (i).

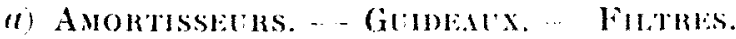

A l'amont du hatleur est amcinagia unc plage en entochements destince a absorber la houle secondaire émise à lopposi de la loule principale. Lal houle émise par le batteur passe a travers un groupe de fillres du type a toles perforces (fis. 3). Elle "st suidé, dans le comvergent, par une série de suideaux metalliques lonsiludinatux, qui empichent les balancements

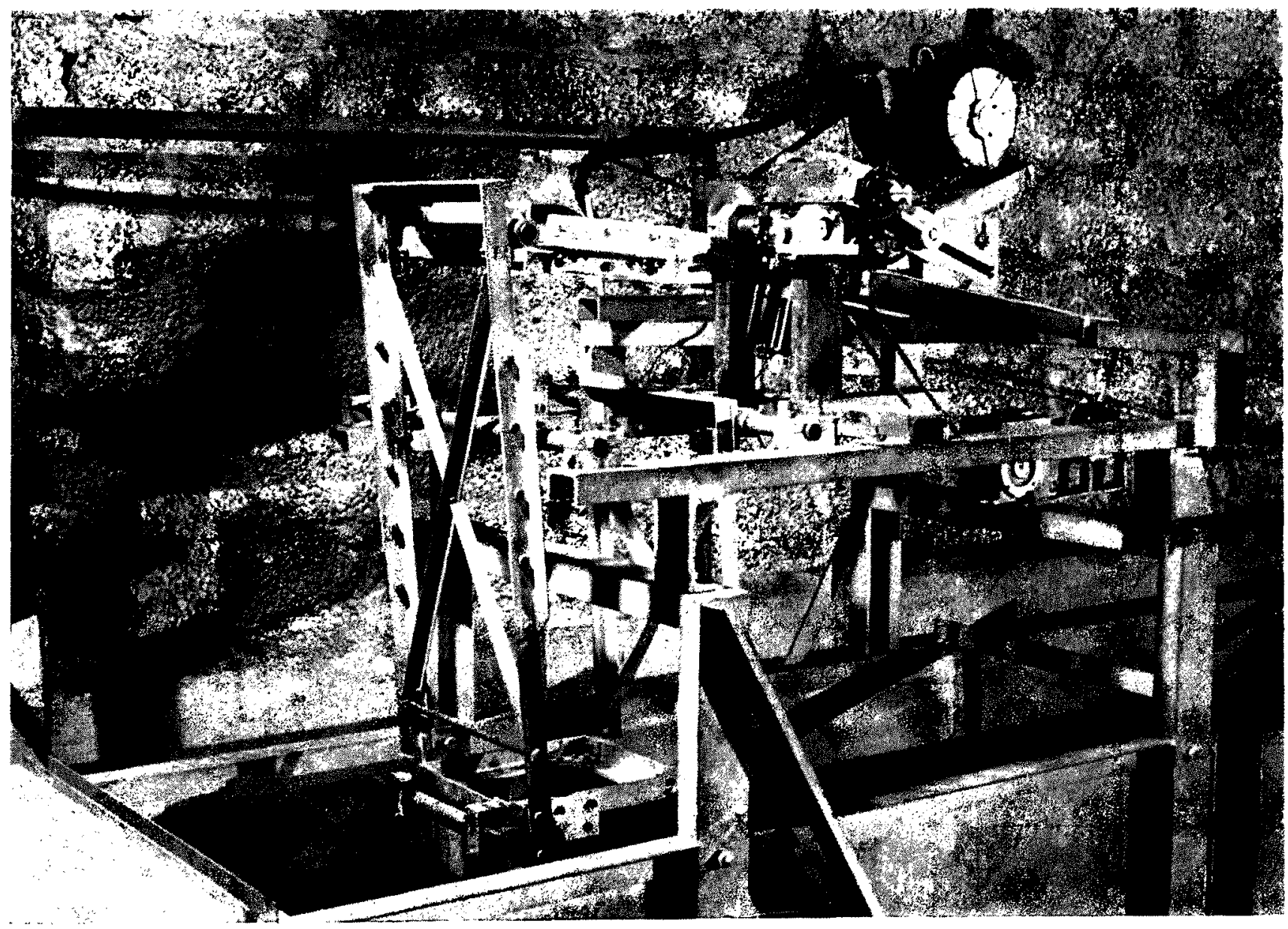

Fici. . . Mecanisme de commande du batkur. 


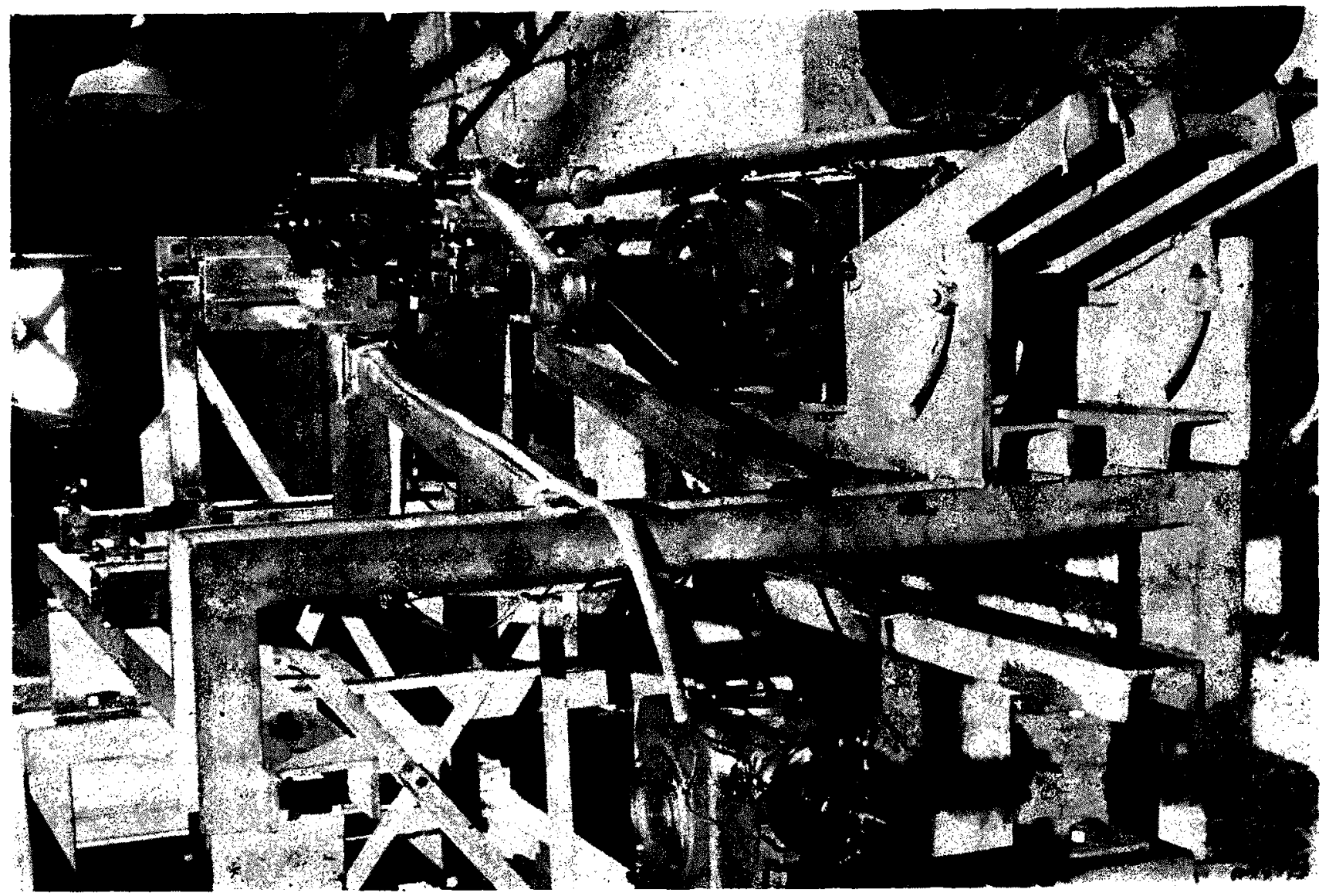

Fu. 6. -.. Mecanisme de commande du batteur. Détail.

transversaux qui pourraient se produre pour cerlaines périodes.

Apres ces guideaux, la houle traverse encore des filtres constitués par un ensemble de feuilles de grillage à larges mailles, pliées et soudées costre elles.

A l'extrémité aval le canal possede un amorlisseur destinc a absorber la houle qui arrive, de laçon que la réllexion soit en tout cas négliscable. Cel amortisseur est constituc par unc plage permáable de srande longueus précedée de fillies. Les expériences réalisées sur le pouvoir réfléchissant des plages perméables ont permis d'établir le profil et la longueur les plus convenables. Pour une plage curviligne de $5,6 \mathrm{ml}$ de longueur, le pouvoir réflichissant reste, quelle que soit la longueur d'onde, de l'ordre de $7 \%$ (selon A. WALLIT).

\section{h) PASSAGL IN CHARGE.}

Le passage en charge a cté placé dans l'élément vitri du eanal ì houle, ce qui donne des longueurs convenables pour les deux hiefs. On appellera bief amont celui qui est du côté du batteur. Le troncon en charge est limite par une sorte de caisse, dont les parois sont assemblées avec un léger jeu. Les trois parois parallèles à Ia direction générale du canal sont en bois, les deux parois normales à cette direction sont en duralumin. Les parois latérales sont appliquées contre les vitres du canal au moven do barres fileties avec des acrous. I.e toul peul se deplacer en hauleur, rendant le passage plus ou moins important (fig. 2). La longuemr du passage 'n charge a été successivement $58,3-116,7$ et $175 \mathrm{~cm}$, elle a done varic dans les rapports 1,2 a 3. La largeur était celle du canal, e'est-àdire de $0,30 \mathrm{~m}$.

\section{II. - Appareils de mesure}

a) Mrsuke de ta période.

La période a été mesurée en comptant les lours de la manivelle du batteur pendant un temps contrôlé au moyen d'un chronomètre.

\section{b) Mrstirf: dis Ampltrubls.}

Pour la mesure des amplitudes du mourement 
oscillatuire de la surface libre on a emplove des pointes de mesure at $1 / 10^{\circ}$ de $m m$, avee indicateur a oeil cathodique. Ayant vérifić que la lecture inférieure étail affecté d'une erreur due a la tension superficielle, on a ajoule une deuxieme pointe recourbe aver haquelle on a fait lobservation directement à l'oil. La comparaison entre les lectures faites directement et avec l'oil rathodique a montré, pour des amplitudes entre 1 ef $30 \mathrm{~mm}$, une erreur systémalique par defaut pour l'oeil cathodique allant de 0,6 a $1 \mathrm{~mm}$ (voil lablealu I).

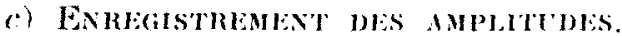

On a employe l'enregistreur d'ondes Neyrpic. Cet appareil enregistre les indications de sondes a caplacitc. Lat sonde est constiluce par un support en acier sur lequel on monfe plusieurs spires d'un fil conducteur convenablement isole. La variation de capacite due à la montíe ou descente de l'eau est transmise à un bloc dectronique qui agit sur un enregistreur Pikly. où les variations du niveau restent inseriles sur une bande de papier yui se dereule à une ceretainc vilesse.

\section{CHAPITRE III}

\section{RECHERCHES EXPERIMENTALES QUALITATIVES}

Nous avons fait, d'abord quelques essais pour avoir une ide des caracléristigues fondamentatles du phénomene produit. Au cours de ces essais on a fait varier la période du batteur et l'enfoncement de l'obstacle.

Nous avons constati ainsi qu'une houle parlant du batteur donne lieu à un clapotis partiel a lamont du passage en charge, à un mourement oseillatoire en masse dans celui-ci of i une houle dans le bief aval, lacpuelle est absorbé par la plage amorlisseuse. Cette houle présente une amplifude decroissante quand on diminue la piriode et quand on augmente renfoncement de lobstacle.

Pour mieux conmailere les détails du mouroment, nous avons mis en suspension des goutfeleltes d'un melange de benzine of de frichlo-

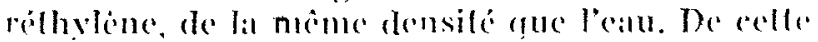
facon, on a bien mis en ćvidence loseillalion en masse dans le passage en charge. Nous arons moiecte sur un papier audrille l'ombre de goultelettes, er crui nous a permis une estimation de l'amplitude de l'oscillation en masse. Ic ranport entre cette amplitude el l'amblitude de la hould transmise (houle a l'aval de l'obstacle) est variable ave la période.

Nous arons constaté aussi un dícollemenl du courant sur les extrémites de lobstacle, alternant avee le mouvement. Pour dablir une comnaraison avec le même phénomène ell régime permanent, nous avons fait couler un rertain débil dans le canal. Les vitesses étaiont du mêne ordre, et même inférieures aux vitesses movenness hI mourement oscillatoire. Ia zone affectán par la dieollement est plus citendue dans la cas do régime permanent.

\footnotetext{
I'nbscrvation du mouvement des goullelelles
}

dams le massage an charge amsi que orlle des valiations des niveaux a l'amont of a lavat, nous a permis de vérifier l'existenee d'un déphasage. Le déplasage cntre l'oscillation amont ef l'oscilhation on masse est variable, en sins, entre - 1, T a t T. L'oscillation a laval est a peu pres en quadrature avee loscillation en masse. Bien entendu, il s'asil d'estimations asse\% impririses.

Pour verifice le déphasage entre loseillation de la surface libre a lamont el a l'aval de lobslacle, nous avons mis sur les deux eotés les dens

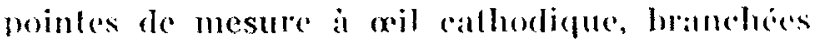
l'une à lautre. Chateune était dans la position de leclure sumericure, de facon a donner les signamx correspondants au maximum. Ene differenere bat bien visible entre les deux signatux. Il est vai que eetle indieation ne enrespond pas exacte. ment an dephasage colde houle incidente el hould transmise. I'n procedé pour la mesure de ce di-

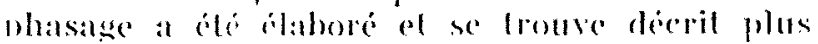
Ioin (chapitre $V$ ).

L'examen des trajectoires à l'amont el à l'aval de l'obstacle nous a montri gue les zones aver forte perturbation sont relativement reduites. A l'amont. a une dislanee de quelfues eentimitres. on peut reconnatre une section où les Irajecloires sont pratiquemenl verticales. Le bremier ventre du clapotis partiel se trouve done un peu à l'amont de la paroi verticale de lobstaicle. Il est vrai que contre celle-ci le mourement est aussi vertical; mais au-dessous it est plutot horizontal; dans son ensemble, le mouvement dans cette section correspondrait, done, à une seclion inlermediate entre un ventre at un neruel du clapotis partiel. On peut done preverie un diplacement des ventres et des noruds du clapotis particl rers l'amont. 


\section{SCHÉNA DU PHÉNOMÈNE.}

D’après les observations qualitatives décrites précédemment, nous avons fait le schéma suivant :

a) A l'amont: houle incidente plus houle réfléchie;

b) Au passage en charge : mouvement oscillaloirc en masse; c) A l'aval : houle transmise,

d) Au voisinage de lobslacle: deux zones perturbées dont l'extension est relativement petite.

Ce schéma du phénomène a été à la base de la théorie et du programme des expériences ct des mesures.

\section{CONSIDERATIONS THEORIQUES}

\section{Nomtroxs (roir lig. 2).}

b largeur du canal.

$h$ profondeur d'ean.

$l$ longueur de l'obstacle $S$.

$h^{\prime}$ hauteur du passage en dessons de l'obstacle.

$a, 0$ amplitude et phase du mouvement dans le passage en charge.

$a_{1}, b_{1}$ amplitude et phase de la houle incidente $(x=0)$.

$a_{2}, f_{2.2}$ amplitude et phase de la houle transmise $(\boldsymbol{x}=\boldsymbol{l})$.

a:, $0_{3}$ amplitude et phase de la houle réflechic $(x=0)$.

$A_{i}=a_{i} e^{i(k t+\theta)},(t=$ temps $)$.

T période du mouvement $(k=2 \pi / \mathrm{T})$.

I. longueur d'onde $\quad(m=2 \pi / \mathrm{L})$.

$a_{2}=t$ rapport des amplitudes de la houle transmise et de la houle incidente.

$\frac{a_{3}}{a_{1}}=r$ rapport des amplitudes de la houle réftéchie ef de la houle incidente.

o, déphasage entre houle transmise ef houle incidente.

ơ déphasage entre houle réfléchie et houle incidente.

c coefficient de résistance linéaire.

$c_{1}$ coefficient de résistance quadratique.

$P$ pression.

$\Psi$ potentiel des vitesses.

- masse spécifique superficielle du liquide.

Nous allons tenter de construire une théorie approche et, d'ailleurs, semi-empirique du phénomene. Elle a pour objet non pas de fournir le potentiel de vitesse de la masse liquide en mouvement, mais seulement de donner des expressions approximatives des rapports $t$ et $r$ pour une configuration donnée des parois rigides. C'est là d'ailleurs le problème essentiel pour l'ingénieur.

Nous partirons des hypothèses suivantes basées sur l'observation du phénoméne dans le canal à houle.

a) Au-dessous de l'obstacle le mouvement du liquide est très roisin d'une oscillation harmonique en masse. Cela nous permet de le représenter au moyen du potentiel :

$$
\Phi=-a k x \sin (k t+0)
$$

en admettant que le mouvement est monopériodique sinusoïdal avec des vitesses horizontales :

$$
\Phi=\mathrm{A}(x, y) \sin \left(\frac{2 \pi}{\mathrm{T}} t+0\right)
$$

Done :

$$
\frac{\partial \mathrm{A}}{\partial y}=0 ; \quad \Delta \mathrm{A}=\frac{\partial^{2} \mathrm{~A}}{\partial \mathrm{x}^{2}}=0
$$

A $(x, y)$ est bien une fonction linciare de $x$.

b) A l'amont de l'obstacle $S$, le mouvement semble être de nature tris complexe. Dans le voisinage immédiat de $S$ il ne parait pas possible d'analyser le phénomène, mais la zone affectée par le mouvement désordonné est réduite. La zone de raccordement entre le mouvement dû aux houles et le mouvement en masse est une tranche de faible épaisseur par rapport à la longueur d'onde et à la longueur de l'obstacle. En première approximation, les forces d'inertie correspondantes paraissent pouvoir être négligées. Dans la zone amont de l'obstacle jusqu'aux filtres, le mouvement du liquide semble être representable par la superposition de deux houles progressives de Stokes, de même longueur d'onde 
se propageant en sens contraires, et d'amplitudes respectives $a_{1}$ (houle incidente), et $a_{: 3}<a_{1}$ (houle réfléchie).

c) A l'aval de l'obstacle, à une faible distance de $S$, on peut admettre qu'il y a déjà une houle progressive de même période que la houle incidente (done de même longueur d'onde). Cette houle sera appelée houle transmise of nous noterons $\alpha_{2}$ son amplitude.

d) Nous admettons que les vitesses et les amplitudes sont faibles, de telle sorle que les équations du problème seront linéarisces.

Il faut noter que les hypotheses faites sont incohérentes du point de vue mathématique. Fn effet, le potentiel $\Phi(x, y, t)$ de la masse liquide en mouvement est une fonction analytique dans fout le domaine occupé par le liquide. Or, nous altribuons a is des formes analytiques fort différenles dans chacune des zones considereses ci-dessus, atueune de ces formes n'étant le prolongement analytique des autres.

Rappelons la formule classique du potentiel des vitesses dans le cas d'une houle de Srokss :

$\Phi_{i}(x, y, t)=$

$$
-a_{i} \frac{k}{m} \frac{\operatorname{ch} m u}{\operatorname{sh} m h} \sin \left(k h \pm m x+0_{i}\right)
$$

$i=1,2,3$, correspond respectivement à : houlc incidente, transmise et réfléchie. On prend le signe + pour $i=3$ et le signe -- pour $i=1$ el $i=2$.

L'origine $O$ des coordonnces est prise à l'intersection du fond du canal avec la face amont de $S$; on est oriente dans le sens de propagation de la houle incidente.

L'équalion de la surface libre pour une houle est:

$$
y=h-h-a_{i} \cos \left(l i t-m x+0_{i}\right)
$$

Comple tenu de l'éfuation:

$$
g !++\frac{\mathrm{P}}{\varphi}+\frac{\partial \omega}{\partial t}=0
$$

et en faisant la pression $P=0$ à la surface libre, on obtient pour la pression :

$$
\begin{aligned}
\mathrm{P}_{i}= & g(h-y) \\
& +g a_{i} \frac{\operatorname{ch} m y}{\operatorname{ch} m h} \cos \left(l_{i} t-m x+0_{i}\right)
\end{aligned}
$$

pour une houle.

Appliquons maintenant les hypotheses faites au $\$ 2$; nous pouvons négliger la zone définie a l'alinéa $b$, appliquer le théorène du mouvament du centre de gravité à la masse oscillant sous l'obstacle et écrire l'équition de continuité en supposant que les formules dus 4 sont valables jusqu'à l'obstacle. A noter que ceei est faux an point de vue mathematique, ear les conditions aux limites ne sont manifestement pas remplies.

A la rérité, nous pourrions raisonner encore de la manière suivante: appelons $\varepsilon^{\prime}$ l'épaisseur de la zone de racordement amont, el é l'épaisseur correspondante aval. Les formules ci-ilpries seraient applicables pour les sections d'abseisses $-\varepsilon^{\prime}$ et $\left(l+\varepsilon^{\prime \prime}\right)$, les forces d'inertio des deux zones etant negligeables. Il en resulte que les formules ultérieures seraient valables moyennant une modification convenable de la valeur $g_{i}(i=1,2,3)$.

D’apres (5), la poussée subie par le liepuide audessous de l'obstacle s'éril (les efforts dus a la pression hydrostatique a l'aval of at lamont se compensent) :

$$
\begin{aligned}
& \mathrm{E}==g / a_{1} \cos \left(k i t+0_{1}\right)+a_{33} \cos \left(k i t+\theta_{33}\right) \\
& -a_{2 .} \cos (k t+0.2) 7 \int_{0}^{h^{\prime}} \operatorname{ch} m ! ! n d !
\end{aligned}
$$

(111 :

$$
\mathrm{E}=\& g \frac{l}{m}, \frac{\operatorname{sh} m h^{\prime}}{\operatorname{ch} m}
$$

$\left[a_{1} \cos \left(k t+0_{1}\right)+\operatorname{ars} \cos \left(k t+0_{3}\right) \ldots a_{2} \cos \left(k t+\theta_{0}\right)\right]$

Le produit (masse $x$ accoléralion), pour la masse oscillant dans le passage en charge, est:

$$
-\cdots l h^{\prime} a k^{2} \cos (k l+9)
$$

Les perturbations créces par l'obstacle, principalement au voisinage des coins, sont difficiles a analyser exactement. Nous admettrons que la présenee de lobstacle se traduit par me risislance horizontale proportionmelle a la vilesse de loseillation en masse. Doù une résistance du type :

$$
-\cdots g h^{\prime} c \frac{\partial x}{\partial x}=g h h^{\prime}(n k \sin (k l+0)
$$

Ic coefficient c ctant une fonction inconnue, a priori, des parandies $h, h^{\prime}, l$. De telle sorte que l'équation dynamique s'écrit :

$$
a_{1} \cos \left(k t+a_{1}\right)+a_{3} \cos \left(k t+a_{3}\right) \ldots a_{2}\left(\cos k t+0_{2}\right)
$$

$a m h^{\prime} f\left\lceil\left(\frac{l k^{2}}{g}\right) \cos (k l+0)+k c \sin (k \cdot l+0)\right\rceil$ 
ayaul latil. pour simplifier :

$$
f=\frac{\ln m h}{\mathrm{dl} m h}
$$

En passant à la forme complexe, nous arons donc l'expression :

$$
A_{1}+A_{3}-A_{2}=A m h^{\prime} l_{g} l^{2}-\ln i
$$

Considérons maintenanl la zone de raceordement à l'amont. Nous obtiendrons l'éfuation de continuite en écrivant que les débits à travers les sections d'abscisses $x=\ldots$ et $x=0$ sont les mènes. Cela revient a negliger les variations de la masse dues à l'oscillation de la surface libre.

Moyennant les approximations décrites didessus, ie débil des houles incidente el reflechic vaut :

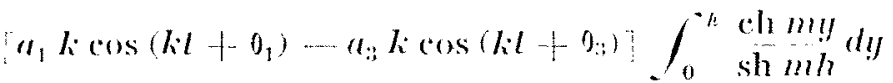

$$
=a_{1} k \cos \left(k t+0_{1}\right) \cdots-a_{3} k \cos \left(k t+0_{3}\right) \frac{l}{m}
$$

Le debil du mouvement en masse vaut :

$$
\ldots h^{\prime} n k \sin (k t+0)
$$

D'où l'équation de continuite:

$$
a_{1} k \cos \left(k t+0_{1}\right) \cdots a_{3} \cos \left(k t+0_{3 ;}\right)
$$

$\therefore \quad-m h a k \sin (k)+0)$

Un rasonnement loul analogue nous donne, pour le colci aval, la redation:

$a_{2} k \cos \left(k t+0_{2}\right)=-m h^{\prime} a k \sin (k t+0)$

Et, sous la forme complexe :

$$
\begin{aligned}
A_{1}-A_{3} & =m h^{\prime} \mathrm{A} i \\
A_{2} & =m h^{\prime} \mathrm{A} i
\end{aligned}
$$

D'où une première relation intéressante :

$$
\mathrm{A}_{1}=\mathrm{A}_{2}+\mathrm{A}_{3}
$$

Du système des équations (11), (16) et (17), on obtient :

$$
A_{2}=\frac{1}{1+\frac{\pi c f}{\mathrm{~T}}+\frac{2 \pi^{2} l f}{g \mathrm{~T}^{2}} i} A_{1}
$$

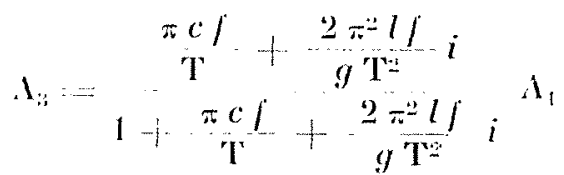

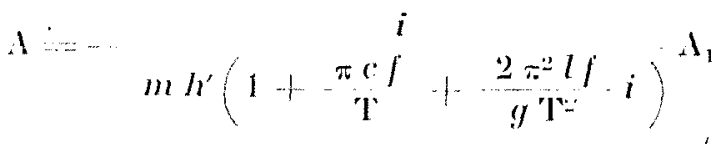

Des equalions (19) ol (20) on pout oblenir les rapports $t$ et $r$.

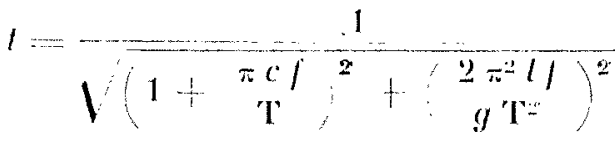

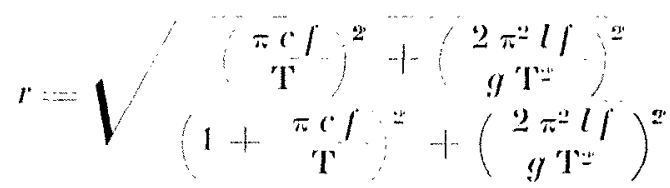

Pour le déphasage entre la houle transmise al la houle incidente, on a :

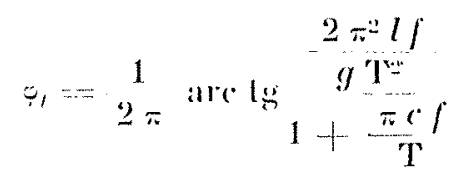

of pour le déphasage entre la houle réfléchie et la houle incidente:

$$
\frac{1}{2} \operatorname{arc} \frac{2 \pi l}{\cos T}
$$

Il est inlíressanl, aussi, de calculer le rapport entre la perte d'énergie et l'énergie de la houle incidente, cest-i-dire:

$$
\begin{gathered}
2 \pi c l \\
\left(1+\frac{\pi c l}{\mathrm{~T}}\right)^{2}+\left(\frac{2 \pi^{2} l l^{2}}{g \mathrm{~T}^{2}}\right)^{2}
\end{gathered}
$$

Pour une resistance nulle, $c=0$, on aura (voir figure 7):

$$
\begin{gathered}
l_{01}=\frac{1}{\sqrt{1+\left(\frac{2 \pi^{2} l f}{g \mathrm{~T}^{2}}\right)^{2}}} \\
r_{10}=-\frac{2 \pi^{2} l f}{g \mathrm{~T}} \\
\sqrt{1+\left(\frac{2 \pi^{2} l l}{g \mathrm{~T}}\right)^{2}}
\end{gathered}
$$



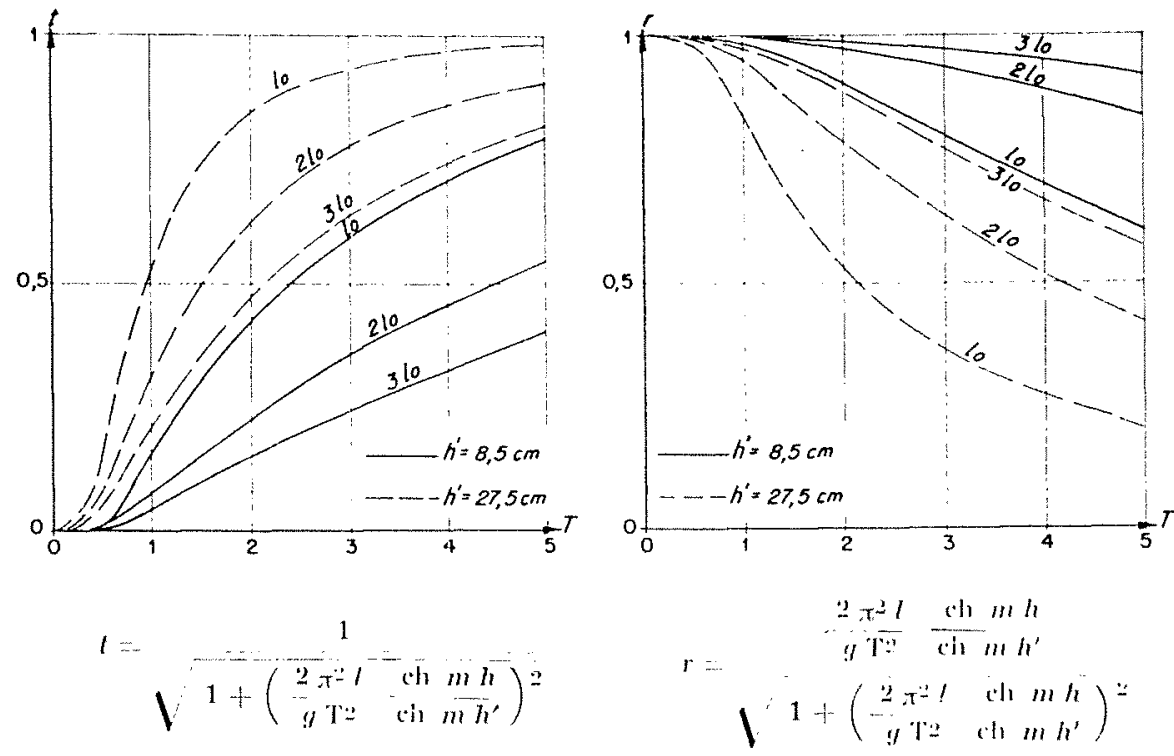

I) 7 ,

La perte relative d'énergie devient alors nulle.

On a, dans ce cas, la relation :

$$
1=l_{1,}{ }^{2}+r_{11}
$$

Les formules (24) el (25) pour les dephasages se réduisent à :

$$
\varphi_{l}=\frac{1}{2 \pi} \operatorname{arctg} \frac{2 \pi^{2} l i}{g \mathrm{~T}^{2}}
$$

el :

$$
\varphi_{r}=p_{1}-\frac{1}{4}
$$

Lat fonction ث̈, est représenté sur la figures.

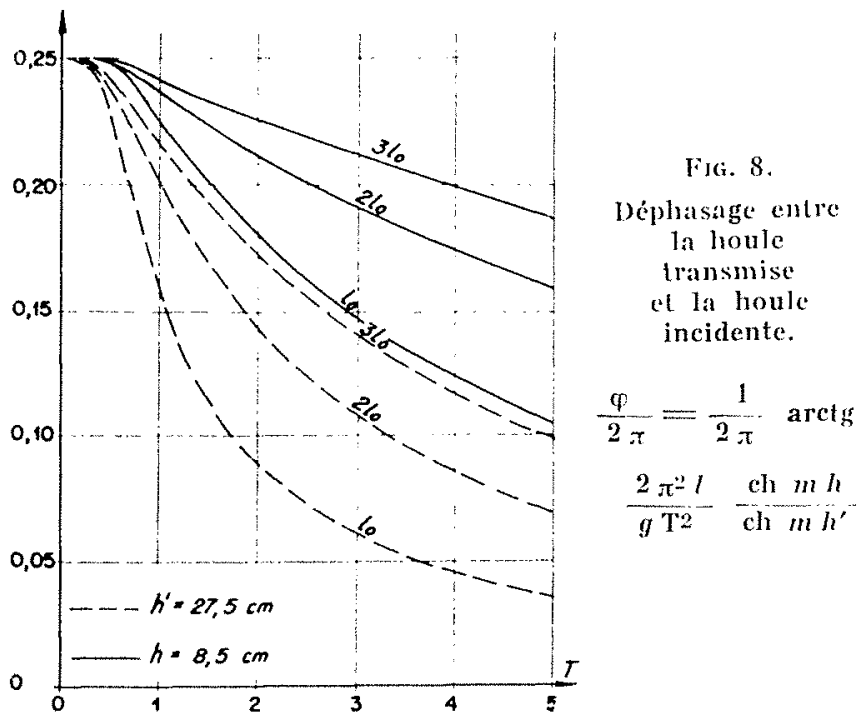

\section{Rosistanch orabitigue.}

Toujours dans le cadre d'un calcul theorique approximatif, on peul essayer de former une solution pour les cas d'une résistance dépendant du carre de la vitesse des oscillations en masse. La formule (8) serait à remplacer par :

$$
? g l^{\prime} \frac{c_{1}}{2 g} a k \sin (k l+0) a k \sin (k l+0)
$$

Comme cette expression est difficile à manier au point de vue calcul, nous lui substituerons, selon te procéde d'approximation bien classique:

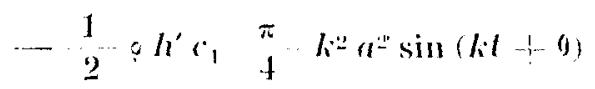

cela revienl, conmon on sait, à remplaces la risistance quadratique par une résistance sinusoïdale de méme période que la vitesse, el de mêne valeur movenne pour une demi-période.

Si on introduit l'expression (33) dans l'équation dynamique, on ohtient, sous la forme complexe:

$$
\begin{aligned}
& A_{1}+A_{*} \quad A_{2} \\
& =-A m h^{\prime}\left(\frac{l l^{2}}{g}-\frac{\pi r_{1} l i^{\prime \prime} a}{8 g} i\right)
\end{aligned}
$$

Les équations de continuité restent les mêmes. On obtient alors :

$$
t=-\frac{1}{\left(1+\frac{\pi_{1} f a_{2}}{g m h^{2} \mathrm{~T}^{2}}\right)^{2}+\left(\frac{2 \pi^{2} l f}{g \mathrm{~T}^{2}}\right)^{2}}
$$




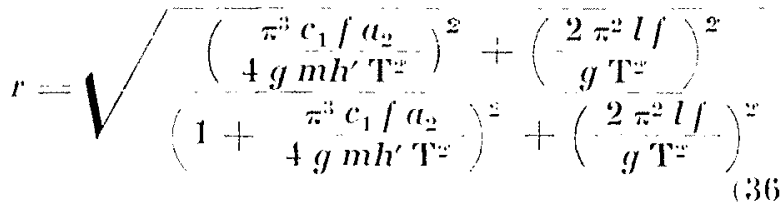

$$
\begin{aligned}
& p=\frac{\pi c_{1} / a_{2}}{+g m h^{\prime} \mathrm{T}}
\end{aligned}
$$

On voit que, dans l'hypothese de la résistance quadratique, $t$ of $r$ dépendraient de l'amplitude de la houle transmise, done de l'amplitude de la houle incidente.

Remarotr. - E... En posant :

$$
x=\pi c i \quad y=\left(\frac{2 \pi^{2} l f}{g \mathrm{~T}^{2}}\right)
$$

Les formules (22), (23) of (26) deviennent:

$$
\begin{aligned}
& t=(1+x)^{2}+y^{2}-1 \\
& r=\left|\begin{array}{c}
x^{2}+y^{2} \\
\left(1+x^{2}+y^{2}\right.
\end{array}\right|^{1 / 2} \\
& p=(1+x)^{2}+y^{2}
\end{aligned}
$$

D'apres leur sons physique, les quantités $p, r, t$ sont essentiellement non nigatives. Mais $c\left(h, h^{\prime}, l, \mathrm{~T}\right)$ est une fonction de forme indéterminée à priori. Cherchons quelle devait être la vatteur de $c$ pour un jeu donné de paramètres $h, h^{\prime}, l, \mathrm{~T}$ (c'est-it-dire pour une valeur fixée de ly), afin que $r$ prenne la plus petite valeur possible et $p$ la plus grande.
On oblient :

pour:

$$
r_{\text {min }}=\left|\begin{array}{c}
2 \vee y^{2}+1 / 4 \\
2 \vee y^{2}+1 / 4+1
\end{array}\right|^{1 / 2}
$$

$$
c=\frac{\mathrm{T}}{\pi f}\left(-1 / 2+\sqrt{1 / 4}+y^{\prime \prime}\right)
$$

la valeur correspondante de $t$ est :

$$
t r_{\text {min }}=\left.\left|2\left(1 / 4+\eta^{2}\right)+\sqrt{ } 1 / 4+\eta^{2}\right|\right|^{-1 / 2}
$$

Si l'hypothese de la résistance linéaire est correcte, on devara done trouver des valeurs expérimentales de $r$ comprises entre $r_{0}$ et $r_{\text {min }}$.

La valeur plus grande possible de plerait :

$$
p_{\max } \cdots\left(1+\sqrt{1}+y^{-1 \cdots 1 / 2}=-\frac{1}{1+i_{0}}\right.
$$

pour :

$$
c=\stackrel{\mathrm{T}}{\pi / 1+y^{2}} \frac{\mathrm{T}}{\pi f t_{0}}
$$

La valeur correspondante de l est :

$$
l_{p \text { miv }}=\left(1-\div \vee 1+y^{2}\right)^{*}+1 y^{2} !-1 / 2
$$

De menc les valeurs de $p$ sont eomprises antre $p=0$ et $p_{\text {main. }}$.

Si on calcule ces quatre fonctions de $y: r_{\text {min }}$, $t_{\text {, min }}, p_{\text {max }}$ el $t_{p \text { max }}$, on peut chereher entre les séries des expériences quelques-unes qui prisentent des conditions favorables pour vérifier expérimentalement l'existence de $r_{\text {min }}$ et $p_{\text {max }}$ en faisant varier la resistance au moyen de filtres platces sous lobstacle.

\section{CHAPI'TRE Y}

\section{PARTIE EXPERIMENTALE}

\section{I. - Mesures \\ (a) PÉRIODE:}

La mesure de la période est simple, et nous l'arons fate au moyen d'un chronometre, plusieurs fois pour chaque essai, soit sur le batteu:, soil sur le mouvement mème de la surface de l'eau en des points convenables.

\section{b) Amplatud:s :}

Nous avons mesuré les amplitudes au moyen de pointes de nesure à weil cathodique. Les amplitudes à l'amont, présentant des maxima et des minima, il fallail pareourir le voisinage de chacun de ceux-ci, surtout pour les minima, pour bien les déterminer. Nous avons mesuré aussi les distances des ventres et des nouds à l'obstacle.

Dans quelques essais, nous avons fait l'enregistrement du mouvement à l'amont grâce à l'enregistreur d'ondes, soit à points fixes, soit en parcourant le camal arec la sonde a capacite transportée par un chariot électrique. Cela nous a montré les détails du mouvement de la surface 
ThIst.AL: I

\begin{tabular}{|c|c|c|c|c|}
\hline$\frac{1+1 \ldots}{\ldots+m e}$ & erme & , er $\frac{1}{h}$ & $A h$ & $\frac{1}{1^{202}}$ \\
\hline \multicolumn{5}{|c|}{$19-8-52=$} \\
\hline $\mathrm{T}=1,2 \mathrm{~s}$ & \multicolumn{3}{|c|}{$l=58,3 \mathrm{~cm}$} & $=27,5 \mathrm{~cm}$ \\
\hline \multicolumn{5}{|c|}{ AMONT(") } \\
\hline & & $\begin{array}{l}\text { becture supe- } \\
\text { rieure lec- } \\
\text { ture inferieure } \\
\text { mm }\end{array}$ & $\begin{array}{c}\text { Ampli- } \\
\text { lude } \\
\text { mm }\end{array}$ & $\begin{array}{l}\text { Distance } \\
\mathrm{cm}\end{array}$ \\
\hline $2 v$ & $1^{\prime \prime r}$ & & $\sim 54$ & 0 \\
\hline $2 \mathrm{~V}$ & 2 & $280,1--224,9$ & 55,2 & 102,5 \\
\hline $2 \mathrm{~V}$ & 3 & $274,5-220,4$ & 54,1 & 187 \\
\hline $2 \mathrm{~V}$ & $f^{*}$ & $279,0-224,0$ & 56,0 & 274 \\
\hline $2 \mathrm{~N}$ & $1^{* 4}$ & $250,4-242,3$ & 8,1 & $5(i, 5)$ \\
\hline $2 \mathrm{~N}$ & $2 n$ & $253,2-241,3$ & 11,9 & 143 \\
\hline $2 \mathrm{~N}$ & 3 & $249,2-238,0$ & 11.2 & 229 \\
\hline $2 N$ & $4^{*}$ & $261,2-241,3$ & 9,9 & 316 \\
\hline
\end{tabular}

- O On ne donne, ici, que les valeurs finales.

AVAL

\begin{tabular}{|c|c|c|c|c|}
\hline $\begin{array}{l}\text { lecture supe- } \\
\text { rieure - lec- } \\
\text { lure inferieure } \\
\text { mm }\end{array}$ & $\begin{array}{l}\text { Ampli- } \\
\text { turle } \\
2 a_{2} \\
m m\end{array}$ & $\begin{array}{c}\text { Distance } \\
\text { em }\end{array}$ & $\begin{array}{l}\text { Lecture } \\
\text { infe- } \\
\text { rieure } \\
\text { Pointe } \\
\text { directo }\end{array}$ & $\begin{array}{l}\text { Lecture } \\
\text { infe- } \\
\text { rieure } \\
\text { Pointe } \\
\text { reconrbie }\end{array}$ \\
\hline $269,6-2+7,8$ & 21.8 & 40 & & 214.1 \\
\hline $254,9-233,7$ & 21,2 & 10.5 & $23.5,0$ & 200,4 \\
\hline $361,0-340.4$ & 20,6 & 1.50 & 341,1 & $30 \bar{T}, 1$ \\
\hline $362,8-3+2,0$ & 20,8 & 170 & 342,7 & 308,7 \\
\hline $36 \mathrm{~T}, 8-3+1,5$ & 20,3 & 190 & $3+2,4$ & 308,2 \\
\hline $362,2-3+2,4$ & 19.8 & 230 & 343,2 & 3049,1 \\
\hline $362,4-342,4$ & 20,0 & 250 & 343,2 & 309,1 \\
\hline $361,6-3+1,2$ & 20,4 & 270 & 341,9 & 307,9 \\
\hline $360,0-340,3$ & 19,7 & 290 & 340.9 & 307.0 \\
\hline $358,5-339,2$ & 19,3 & 330 & 3.10 .1 & 305,9 \\
\hline $357,0-337,7$ & 19,3 & 370 & 338,6 & 301.3 \\
\hline $356,1-336,1$ & 20.0 & 410 & $33 \pi, 0$ & 302.8 \\
\hline
\end{tabular}

VAintas MovenNes :

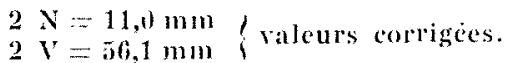

$$
2 a_{2}=20,2 \mathrm{~nm}
$$

DEPHASAGE q $t$

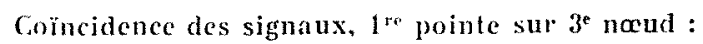

$$
d_{1}=229 \mathrm{~cm} \quad d_{2}=272 \mathrm{~cm}
$$

libre el nous a permis de vérifier si le mouvement était régulier ou non. Les amplitudes des ventres et des nouds ont été controlées avec la pointe à oil cathodique.

L'amplitude de la houle transmise a été mesurée en plusieurs points entre l'obstacle et la plage absorbante. Nous arons fait aussi des enregistrements à points fixes avec l'enregistreur d'ondes, pour vérifier si l'amplitude restait constante et s'il y avait ou non un mouvement de seiche.

Le tableau I, extrait du cahicr d'essais, montre les mesures faites à chaque essai.

Les figures 10 à 13 montrent des enregistrements réalisés avec l'enregistreur d'ondes.

Avant chaque essai on fixe la sonde à capacité sur une pointe de mesure. En faisant varier l'enfoncement dans l'eau au repos, on fait l'bitalonnage (fig. 9). Cel ćtalommage nous permet de fixer l'échelle d'enregistrement el de vérifier la linéarité, el, le cas échéant, den déterminer les icarts.

La figure 10 montre les amplitudes dans la zone d'un ventre. En haut on voit les amplitudes enregistrées en parcourant plusieurs fois la zone du ventre, pour déceler des variations avee le temps. En bas on montre l'enregistrement fait dans le mème but a point fixe. Des enregistrements du mème type ont éte faits pour la houle transmise et avee les deux vilesses de déroulement du papier.

La figure 11 montre un enregistrement avec les deux vilesses de déroulement du papier, pour le clapotis partiel a l'amont, pour $T=0,8$ s, $h^{\prime}=8,5 \mathrm{~cm}, h=30, l=58,3 \mathrm{~cm}$.

La figure 12 montre pour un autre cas $\left(\mathrm{T}=0,8 \mathrm{~s}, h^{\prime}=24,4 \mathrm{~cm}, h=30 \mathrm{~cm}, l=58,3 \mathrm{~cm}\right)$ l'ensemble du phénomene : on y voit, en haut, les ventres et les nouds dus à la superposition de la houle incidente of de la houle rélfichie, el, en bas la houle transmise. D'autres enregistrements correspondent à $\mathrm{T}=1,8 \mathrm{~s}$.

Sur la ligure 13, on a reproduit les enregistrements, en détail, de plusieurs nouds pour montrer la complexité du mourement dans ces zones, oi apparait nettement une oscillation secondaire.

\section{(c) Déphasage o, :}

La mesure du déphasage entre la houle transmise et la houle incidente repose sur te schéma d'un clapotis partiel a l'amont, résultant de la superposition de deux houles sinusoidales. Au droil d'un noeud, le niveau maximum correspond au passage de la crête de la houle. Il faut remarquer que la position des noeuds est, en génciral. bien connue. On place la premiere pointe a ail athodique sur un noud, en général, le troisieme, de façon qu'elle indique le niveau maximum. On mesure la distance $d_{1}$ du noeud à l'obstacle. On 


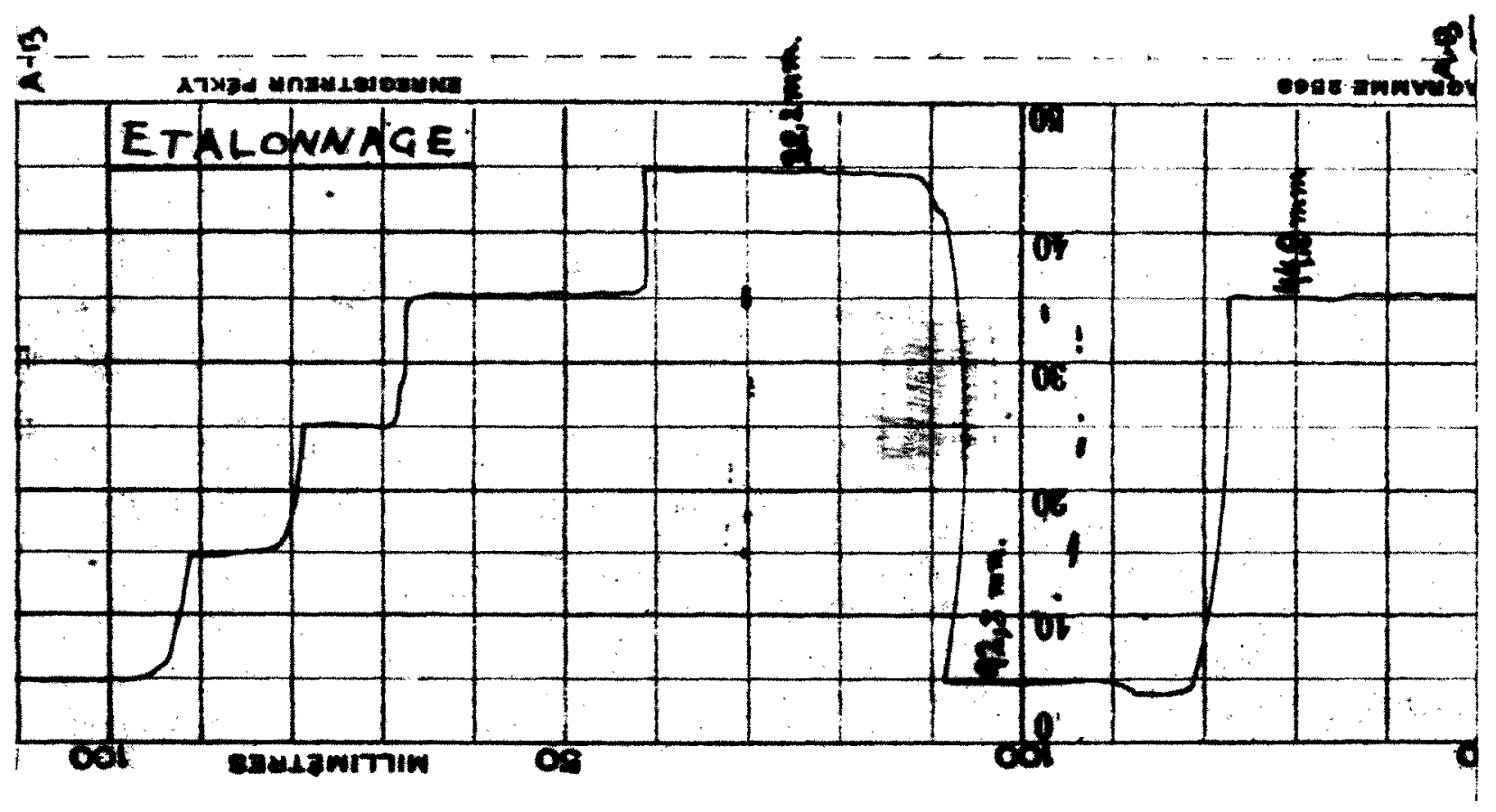

Fisi. 9. -.. L'n exemple d'atalonnage.

laisse celte pointe fixe stu te noud ef on la branche avec la deuxième. Avec cette deuxième pointe on detecte le passage de la houle transmise en differents points, jusqu'à ce que les signaux se produisent simultanément. En réalité, cette simultancité est visible sur un intervalle que l'on determine. On prend, comme distance $d_{.2}$ correspondant à la coüncidence des deux signaux, la moyenne des distance des extrémités de l'inlervalle à l'obstacle.

Avec ces deux distances $d_{1}$ et $d_{2}$ on peut ealculer le déphasage o, comme nous l'expliquons ci-après.

\section{(l) Déphasage or:}

La détermination du déphasage entre la houle incidente et la houle reflechie est aussi indirecte. Pour obtenir ce déphasage, nous avons mesuré les distances de l'obstacle aux ventres et aux nœuds du clapotis partiel.

\section{II. - Interprétation des mesures}

\section{a) AMPlitudes DE LA HOLLE INCIDENTE: ET DE LA HOULE IRÉFLÉCHIE:}

On fait les moyennes des mesures de plusieurs ventres et de plusieurs nouds. Avec ces deux valeurs, on peut calculer les amplitudes des houles composantes. Nous avons employé un abaque préparé au Laboratoire Dauphinois d'Hydrauli- que par M. Cankx, avee les equalions du clapotis partiel au deuxieme ordre. On obtient de cet abarque l'amplitude de la houle incidente et le rapport $r$.

Il faut remarquer que les amplitudes des ventres sont assez grandes el peu différentes. Par contre, les noeuds présentent, en général, des amplitudes petites qui, parfois, ont des différences notables. L'amplitude $u_{\text {, }}$ reste bien déterminée, mais le rapport $r$ est beaucoup moins précis. Des différences de l'ordre du millimetre sur l'amplilude des noeuds donnent des variations importantes du rapport $r$. Par exemple, arec $2 \mathrm{~V}=49,4 \mathrm{~mm}$ et $2 \mathrm{~N}=5,8 \mathrm{~mm}$, pour $\mathrm{T}=1.8 \mathrm{~s}$, on a $r=0,9$ el $2 a_{1}=26 \mathrm{~mm}$. Si $\mathrm{N}=4,6 \mathrm{~mm}$, on obtient $r=1,0$ et $2 a_{1}=24,8 \mathrm{~mm}$; les variations sont respectivement de $11 \%$ et $4,5 \%$.

\section{b) HOILE TRANSMISE :}

Cetle houle présente, en général, une amplitude assez uniforme en dehors du voisinage de l'obstacle el de la zone de la plage ahsorbante. On a fait toujours une dizaine au moins de mesures de l'amplitude à difrérentes distances. Quand les valeurs présentaient des maxima et minima bien définis, on faisait la demi-somme. En cas contraire, on prenait la moyenne de toules les mesures.

Une fois connues $2 a_{2}$ et $2 a_{1}$, on calcule leur rapport $t$. 

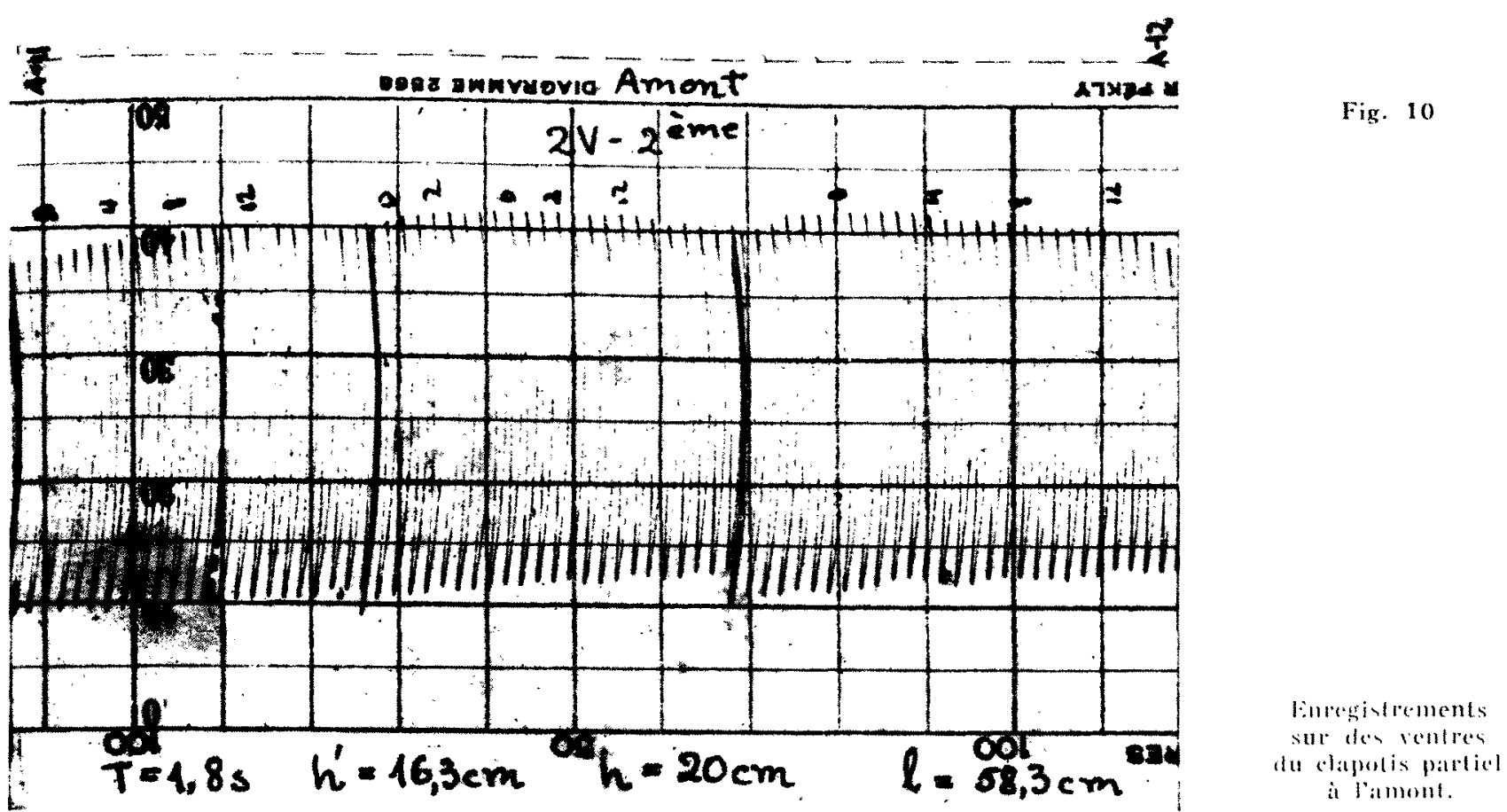

Cithessons :

Entegistrement

de la boule transmise

(2) vilesses

dentergistrement)
10 ecesanmuravia Amont Aาxia un $2 V+2$ enpe. $\quad d=108 c \mathrm{~cm}$

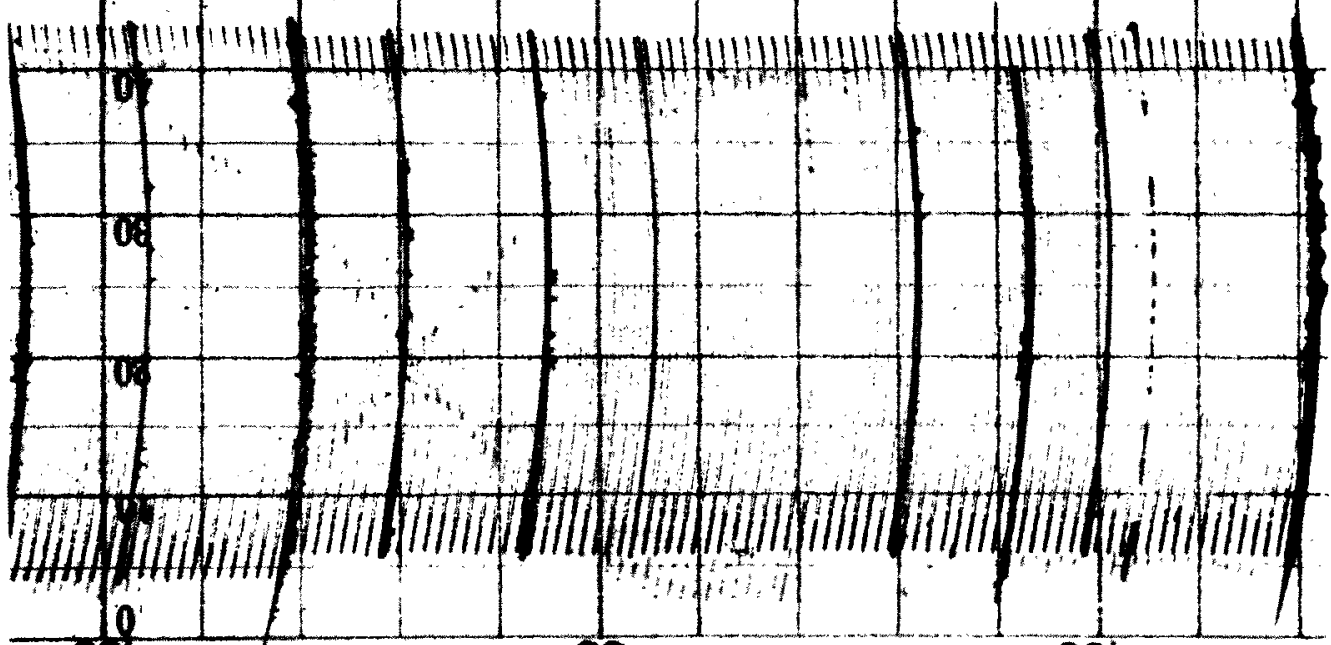

$$
T=1,3 \mathrm{~s} . \quad h^{\phi 9}=24,4 \mathrm{~cm} \quad h=39,0 \mathrm{~cm} \quad 001=58,3 \mathrm{~cm} \text {. }
$$

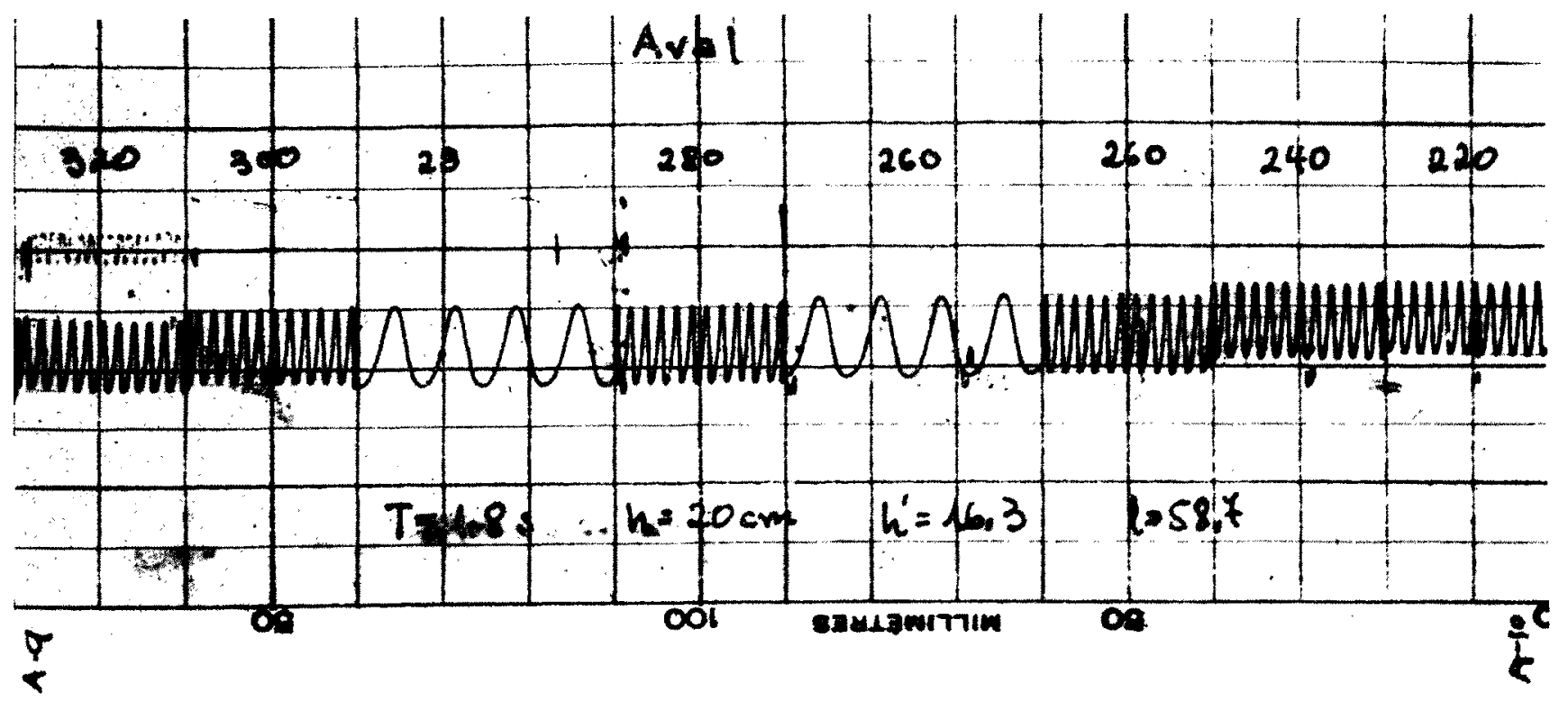




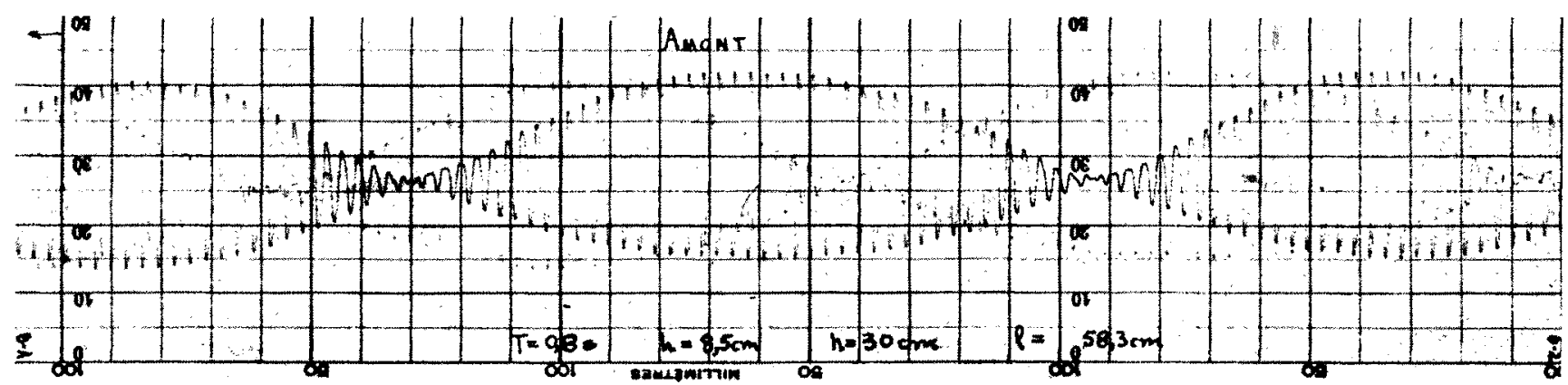

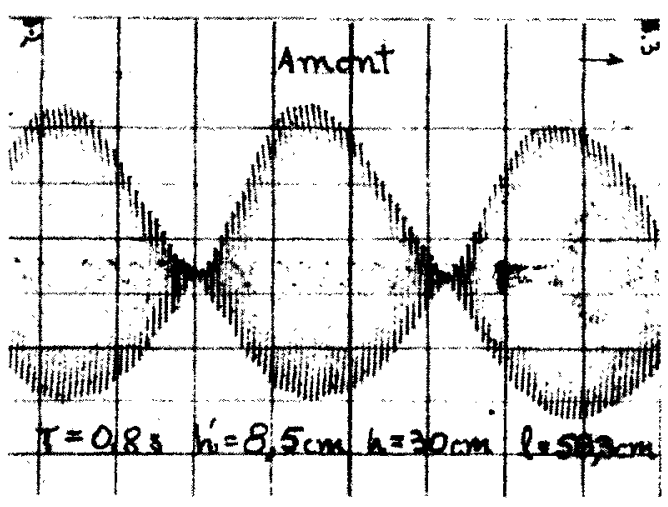

Fig. 11

Enlegistrements de clapolis parliel amont (2) vitesses d'enregistrement).

\section{(c) DÉPHASAGE Q :}

Arec les distancés des différents nouds à l'obstacle, nous avons calcule la longueur d'onde de la houle: L.

Alors, le rapport $d_{1} / \mathrm{L}$ nous donne, en fraction de période, le temps employé par la houle incidente pour arriver a l'obstacle. De même, le rapport $d_{2} / \mathrm{L}$, nous donne le temps qui s'est passé après le départ de la houle transmise. Si la proinle de mesure est placée sur le troisieme noud, le déphasage, en fraction de période, est inal à $2 \ldots\left(d_{1}+d_{2}\right) / \mathrm{L}$.

\section{d) Déphasage $\varphi_{r}$ :}

Pour obtenir ce déphasage, on détermine le déplacement des nouds par rapport aux distances qui correspondraient dans le cas où il y aurait un ventre sur la paroi amont de l'obstacle. Comme ce déplacement est seulement de quel'pues centimetres, les erreurs deviennent importantes.

S'il n'y avait pas un déplacement des nouds, ceux-ci se trouveraient à des distances (2) $n-1) / 4$. I de l'obstacle. Systématiquement, nous avons trouvé des distanees plus grandes. La différence, divisce par la demi-longueur d'onde, nous donne le déphasage or, en fraction de période.

\section{III. - Comparaison avec la théorie}

Nous avons dresse des tableaux donnant les valeurs de $t, t_{0}, r_{\text {miri }}, r, r_{1,}, p, p_{\text {max }}$ en fonction de $\mathrm{T}$, at pour diver'ses dispositions (enfoncement, lonsueur de l'obstacle). $t, r, p$ correspondent ì des valcurs expérimentales. Pour mienx comparer les résultats expérimentaux arec la thérie, nous avons représenté des courbes thériques $r_{0}, r_{\text {min }}$, $t_{01}, p_{\operatorname{mix}}$ sur les figures 14 à 19 . Sur les mèmes figures, on a représenté les valeurs expérimentales. Dans quelques cas, nous avons aussi représenté les courbes pour certaines valeurs du coefficient $c$ de résistance.

Les valeurs expérimentales du rapport $t$ définissent des courbes à peu près parallèles aux courbes théoriques $t_{0}$. Cela nous indique un ecart relatif plus important pour les valeurs plus petites de $t$, c'est-à-dire pour les périodes courtes. D'autre part, les valeurs expérimentales sont plus près des valeurs thériques pour les grandes longueurs et pour des enfoncements réduits de l'obstacle.

II y a une déviation des points expérimentaux par rapport aux courbes theoricues avec résistance. Il faudrait introduire un coefficient $c$ différent pour chaque période. Pour $h^{\prime}=27,5 \mathrm{~cm}$, les valeurs de $c$ devraient varier approximativement entre 0,07 et 0,8 pour $l=175 \mathrm{~cm}$; entre 0,04 et 0,4 pour $l=116,7 \mathrm{~cm}$, et entre 0,03 et 0,3 pour $l=58,3 \mathrm{~cm}$.

On a essayé de calculer le coefficient de résistance avec la formule du mouvement laminaire dans une conduite de section rectangulaire, mais les valeurs qu'on oblient sont complètement négligeables par rapport aux résultats expérimentaux.

Pour avoir une vue d'ensemble, nous avons représenté sur les figures 20 et 21 les fonctions $t_{11}=\left(1+y^{2}\right)^{-1 / 2}$ et $r_{13}=y\left(1+y^{2}\right)^{-1 / 2}$ ainsi que les points expérimentaux correspondants.

Sur la figure 20, les points correspondant à six séries ont été reliés par des courbes. Ces courhes se rapprochent de la courbe théorique du côté gauche, qui correspond à des longues périodes. On y voit aussi que les courbes correspon- 
Fig. 12

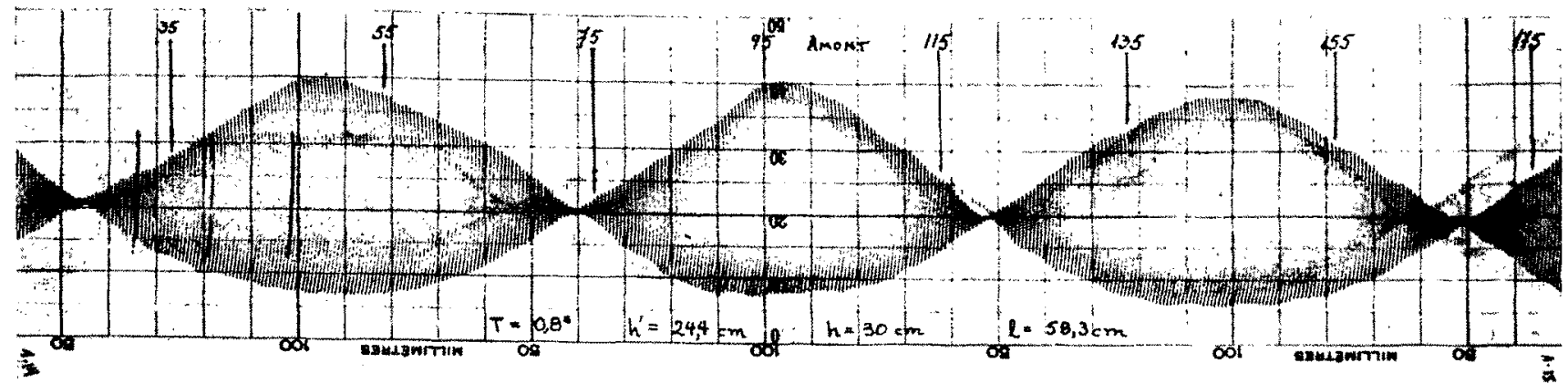

Lc clapotis particl amont.

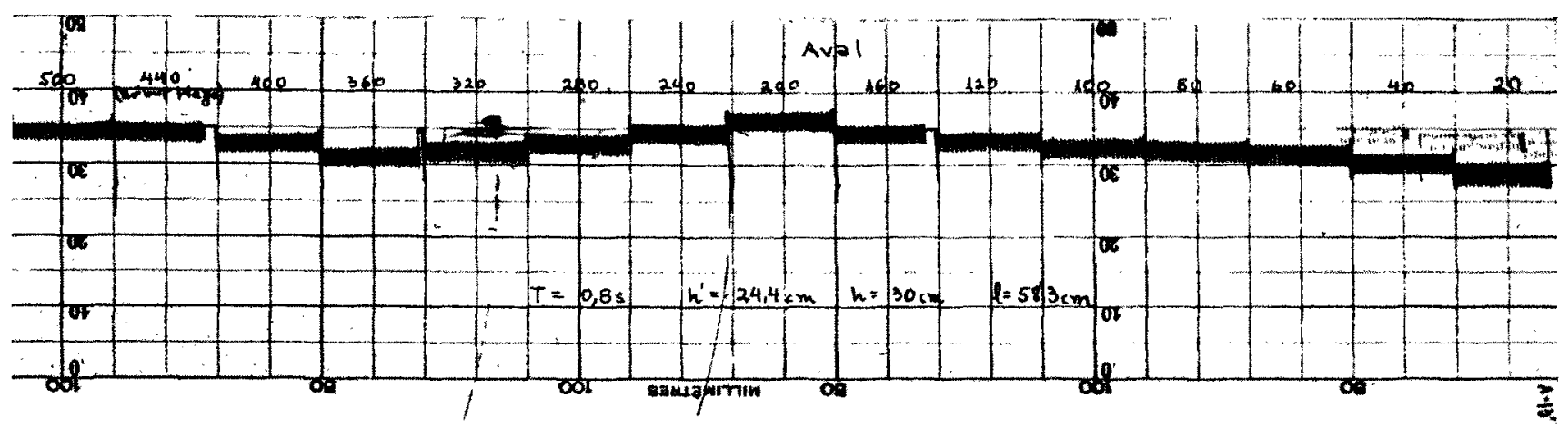

La houle transmise a l'aval.

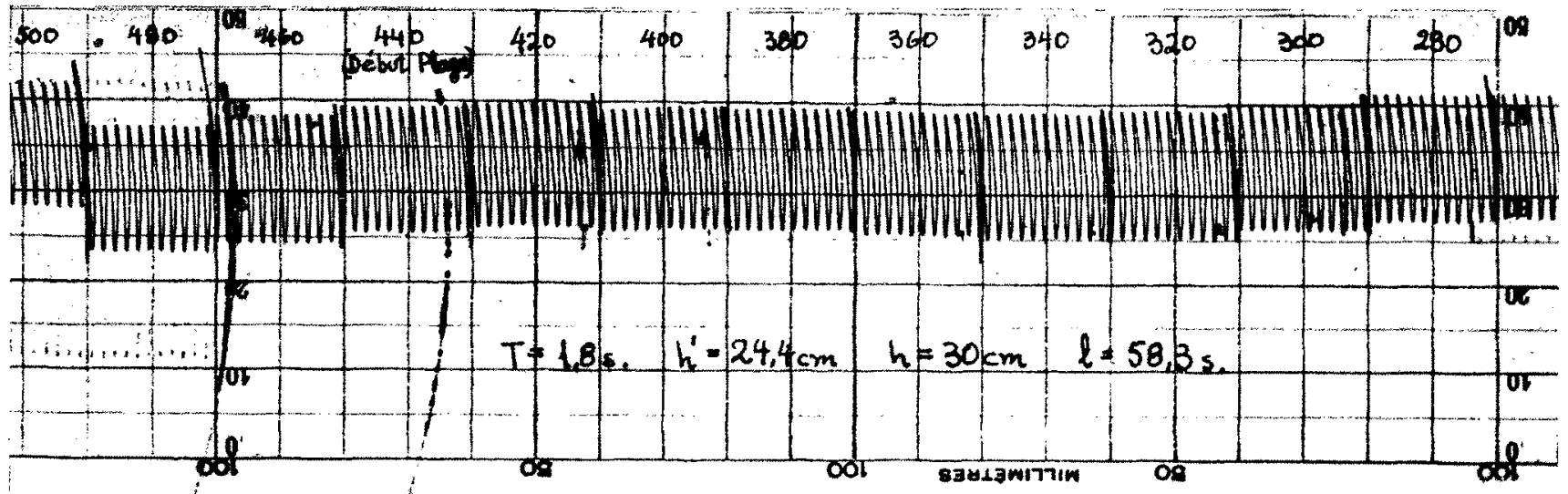

La houle transmise $(\mathrm{T}=1,8 \mathrm{~s})$
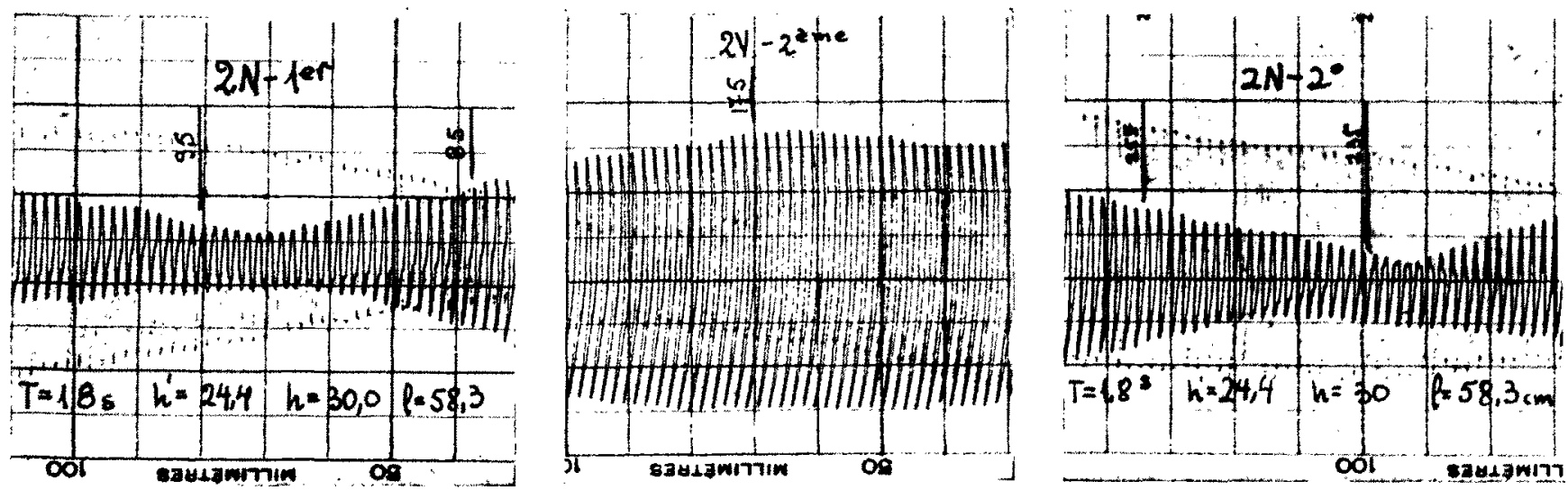

Enregistrements sur les deux premiers nouds et le deuxieme ventre a partir de lobstacle. 

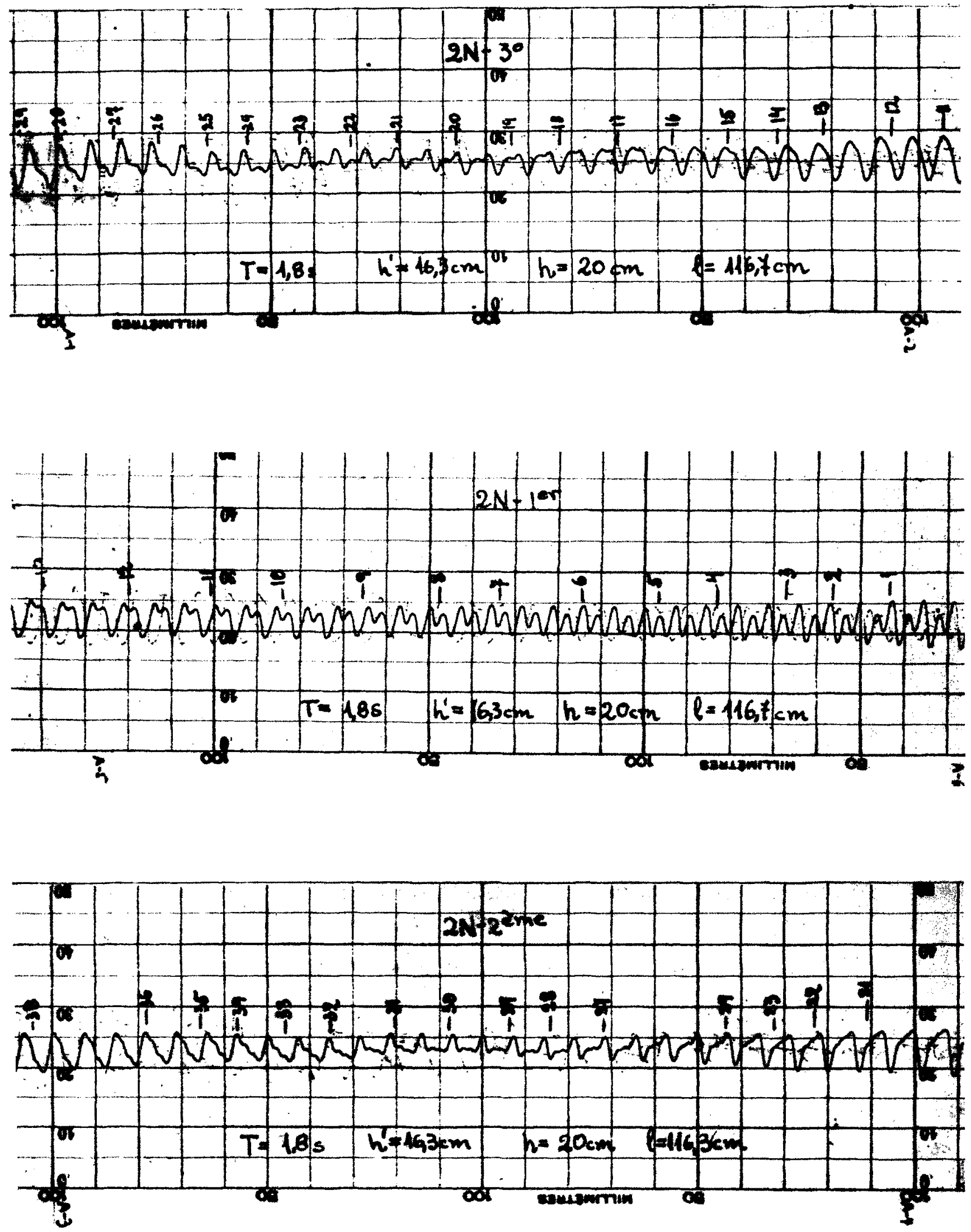
Fig. 13 (suite)
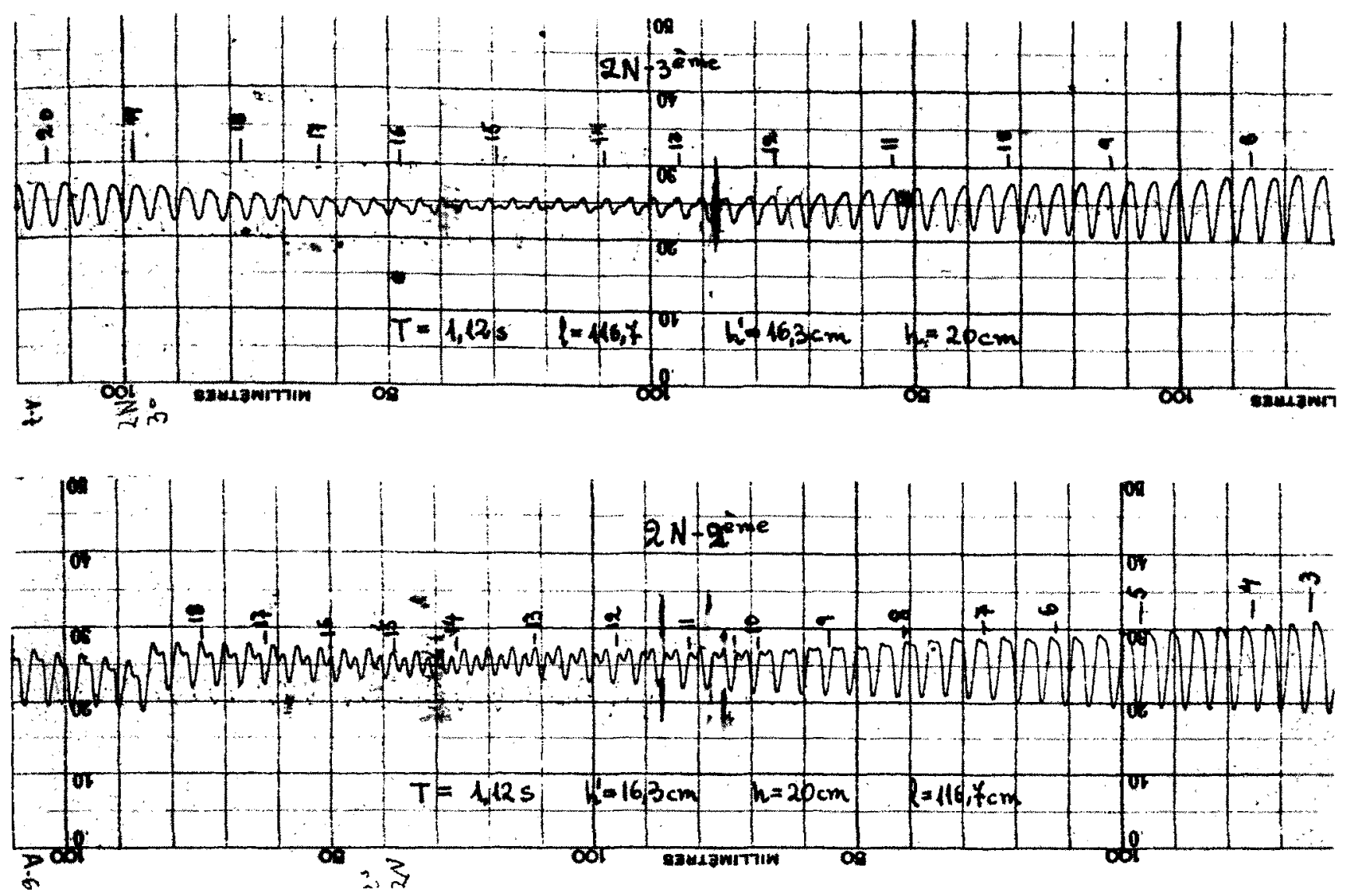

Quelques exemples denregistrement de nouds.
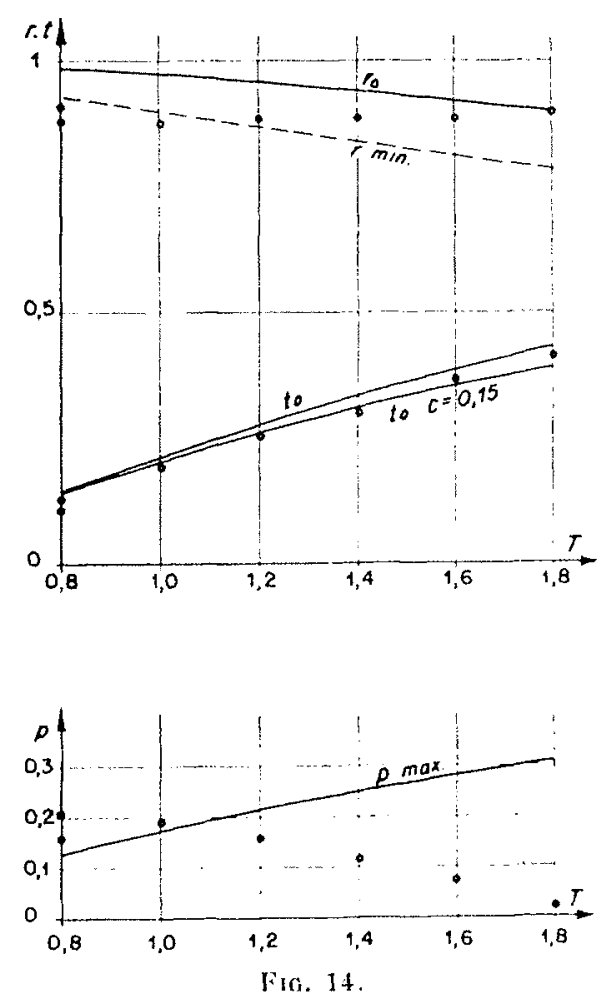

Résultals experimenlaux $(r, t, b)$.

$t=17 \mathrm{~cm} ; \quad h=30 \mathrm{~cm} ; \quad h^{\prime}=27.5 \mathrm{~cm}$ dant aux longueurs plus grandes et aux enfoncements plus pelits se placent plus pries de la (o)the théorique.

Les points experimentaux correspondant au raplort $r$ présentent, en général, nne forte dispersion. Ils sortent friquemment de la bande définic par les courhes $r_{n}$ et $r_{\text {min. }}$. Cette dispersion est ainsi bien risible sur la figure 21 oì nous avons représenle lous les points expérimentaux de $r$.

La dispersion du rapporl r riapparait quand on represente la perte d'énergie relative :

$$
p=1-\left(t^{2}+r^{2}\right) \text {. }
$$

Dans quelques eas, elle presente une allure regulicre, mais on trouve des points qui sortent de la bande delinie par l'axe des abseisses $(p=0)$ et la courbe $p_{\text {max }}$, surtout pour les petites périodes.

Sur la figure $15 \mathrm{~A}$, on a représente les resultats d'une double experience, au cours de laquelle on a étudié le même cas selon deux cehelles. J'application de la similitude de Fnovne nous a permis de la réduire à la mème hase.

Sur les ligures 22-23, on a represente des courbes theoriques des dephasages $t_{0}$ et $r_{0}$, ansi que les valeurs obtenues experimentalement. Pour on a, cu genciral, des valeurs inferieures 

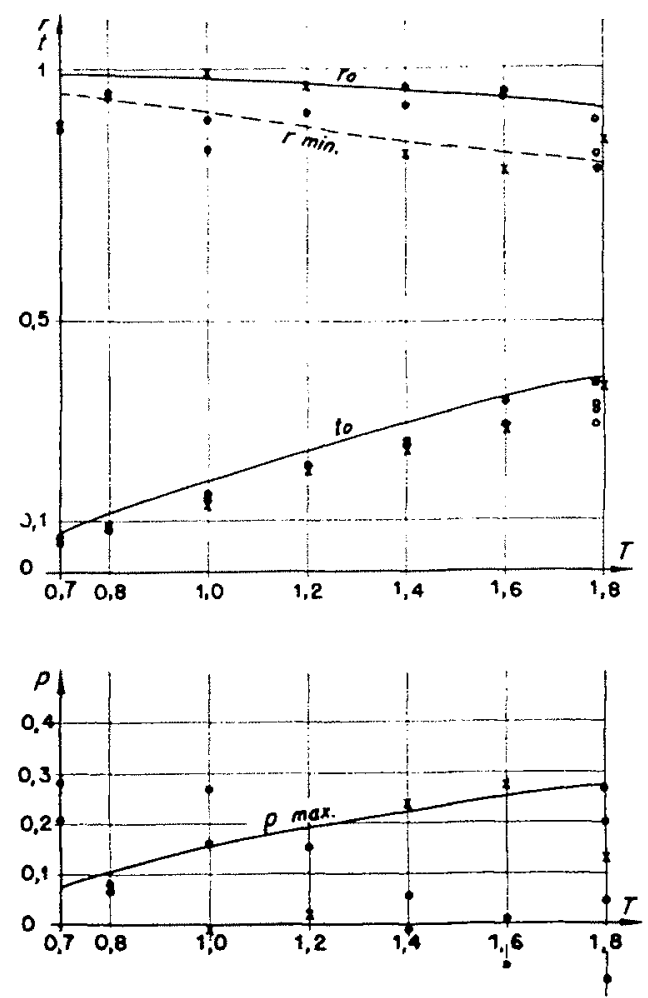

FIG. 15 A.

Résultats expérimentaux $(r, t, p)$.

- $l=175 \mathrm{~cm} ; \quad h=30 \mathrm{~cm} ; \quad h^{\prime}=24,4 \mathrm{~cm}$ $\times l=116,7 \mathrm{~cm} ; \quad h=20 \mathrm{~cm} ; \quad h^{\prime}=16,3 \mathrm{~cm}$

(Ces points ont été obtenus par similitude de Froude.)
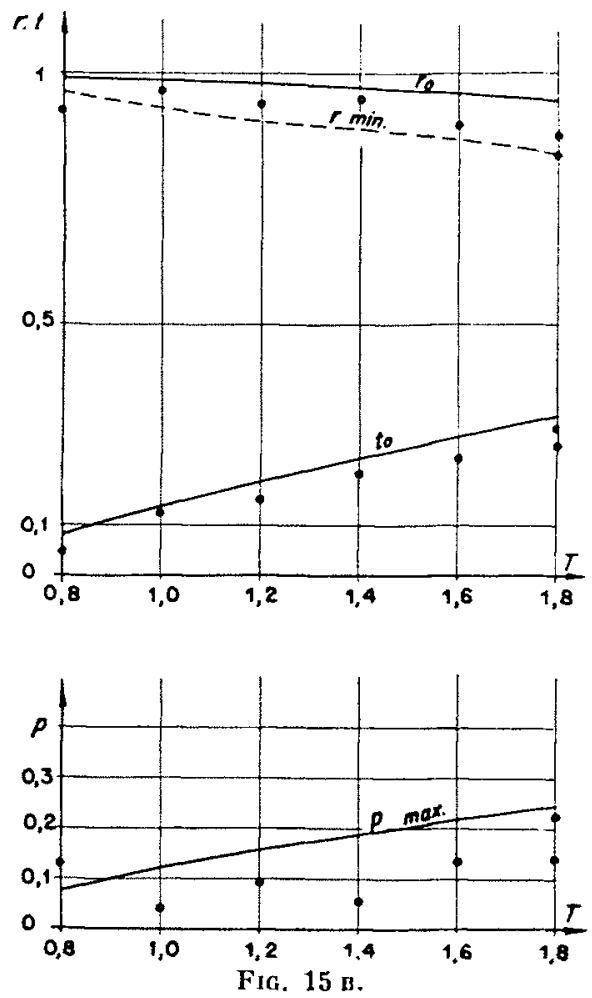

Résultats expérimentaux $(r, t, p)$.

$l=17 \mathrm{~cm} ; \quad h=30 \mathrm{~cm} ; \quad h^{\prime}=19,7 \mathrm{~cm}$
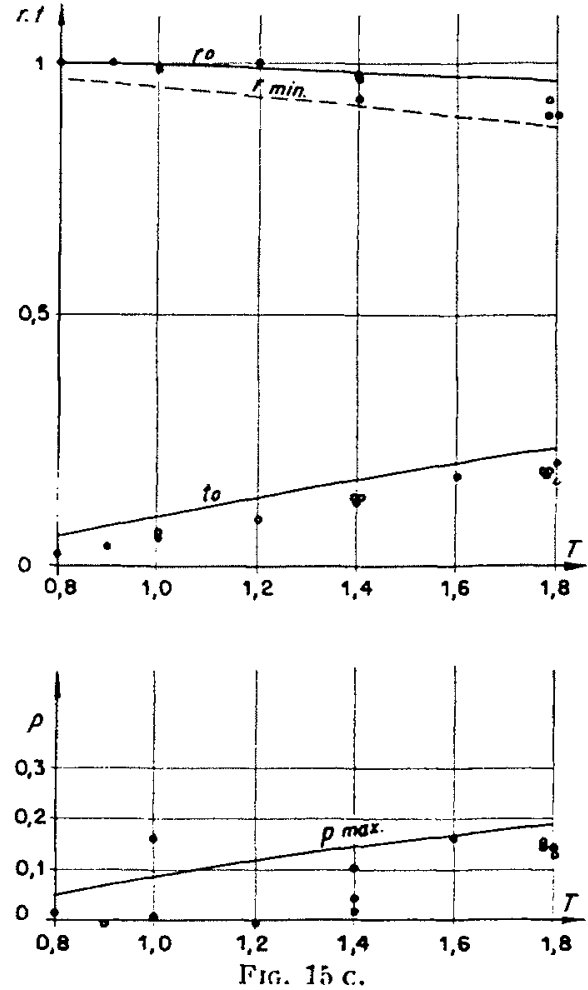

Résultats expérimentaux $(r, t, p)$.

$l=175 \mathrm{~cm} ; \quad h=30 \mathrm{~cm} ; \quad h^{\prime}=15 \mathrm{~cm}$
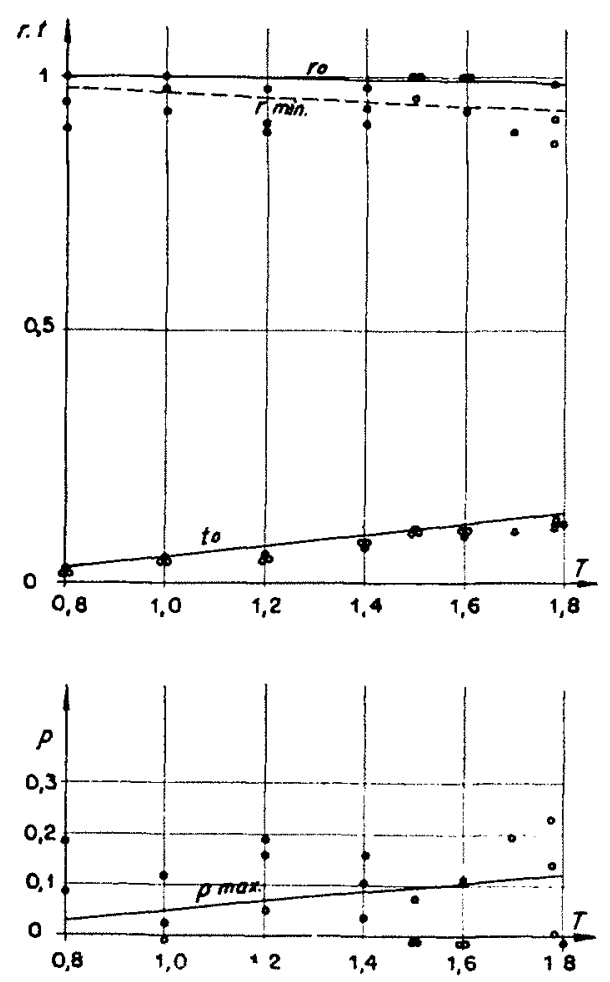

Fig. 16 .

Résultats expérinentaux $(r, t, p)$.

$l=175 \mathrm{~cm} ; \quad h=30 \mathrm{~cm} ; \quad h^{\prime}=8,5 \mathrm{~cm}$ 
Fig. 17
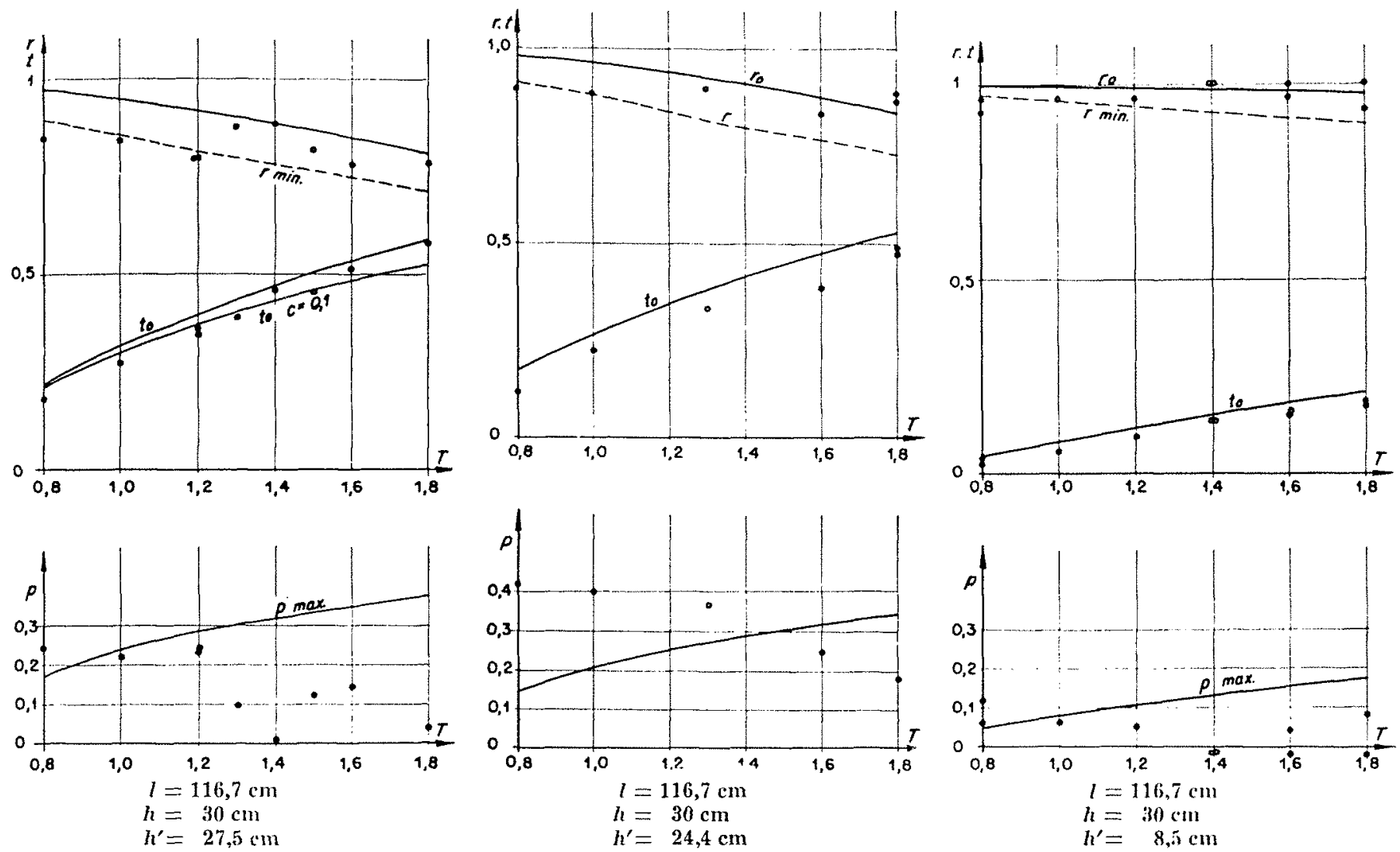

Résullats expérimentaux $(r, l, p)$.
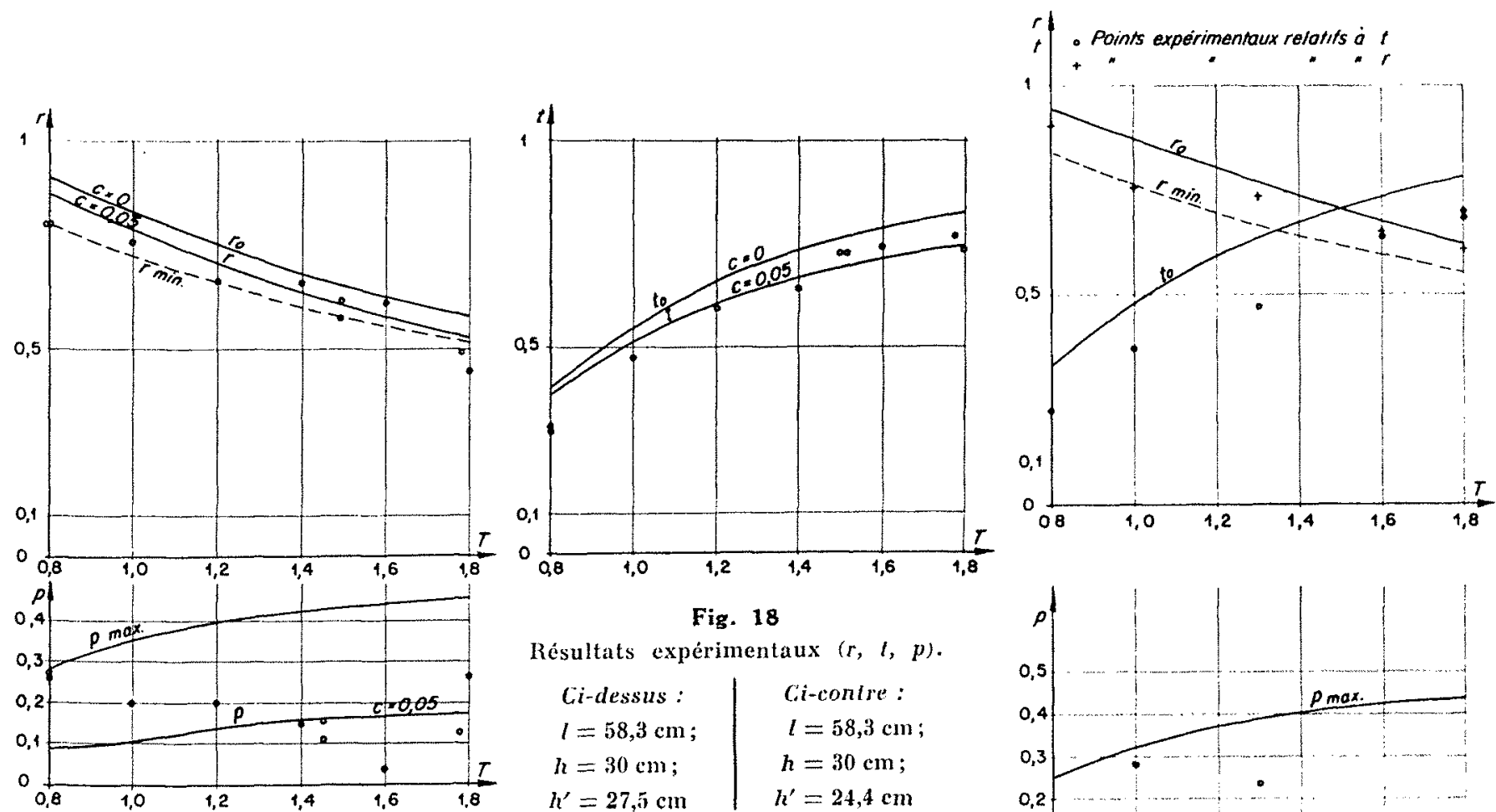

Fig. 18

Résultats expérimentaux $(r, l, p)$.
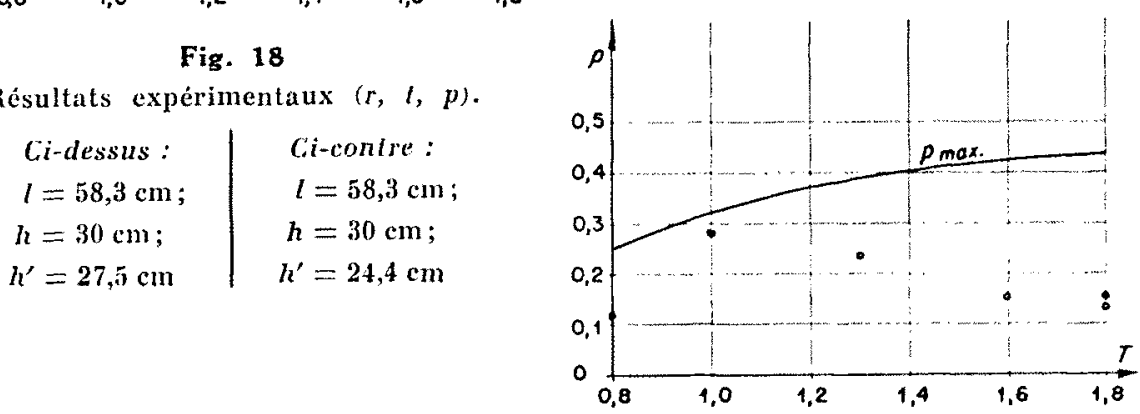
all déphasage théorique † $t_{0}$ sans resistance. Pour , les resultats sont moins bons, il $y$ a une forte dispersion et les points expérimentaux tombent de parl et dautre de la courbe théorique.
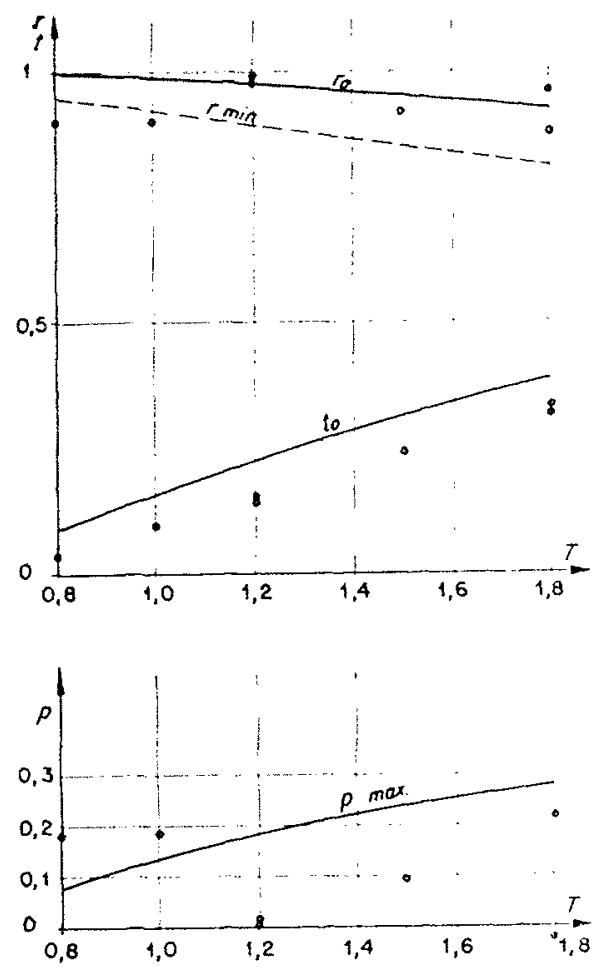

Fig. 19.

Résultats expérimentaux $(l, t, p)$.

$l=58,3 \mathrm{~cm} ; \quad h=30 \mathrm{~cm} ; \quad h^{\prime}=8,5 \mathrm{~cm}$

\section{IV. - Influence de l'amplitude}

Pour étudier l'influence de l'amplitude de la houle incidente sur le phénomène, nous avons fait varier cette amplitude enlre des limites assez ćtendues, au cours d'une grande partie des essais. Le mouvement est moins régulier pour les amplitudes plus srandes, mais on doit tenir compte que cela peut provenir aussi du batteur.

Pour le rapport $t$, l'influence de l'amplitude est assez faible. On lrouve des variations tantôt dans un sens tantôt dans l'autre, comme on peut le constater sur le tableau II gui reproduit une parlie des mesures faites.

En ce qui concerne le rapport $r$, les résultats sont [ro] incertains pour en tirer une conclusion, mais il semble $y$ avoir une augmentation de $r$ avec l'amplitude de la houle incidente. Cette variation est une des causes de la dispersion que présentent les valeurs du rapport $r$ représentées sur les figures.
TABLIAA II

FSSAIS AVLC VARIATION DE L'AMPIITUDE

\begin{tabular}{|c|c|c|c|c|c|c|}
\hline $\begin{array}{l}\mathrm{T} \\
\mathrm{s}\end{array}$ & $\begin{array}{c}l \\
\text { cin }\end{array}$ & $\begin{array}{l}h^{\prime} \\
\mathrm{cm}\end{array}$ & $\begin{array}{l}2 a_{1} \\
\mathrm{~mm}\end{array}$ & $\begin{array}{l}2 a_{2} \\
\mathrm{~mm}\end{array}$ & $t=\frac{2 a_{2}}{2 a_{1}}$ & $r=\frac{2 a_{: 3}}{2 a_{1}}$ \\
\hline 1,2 & 58,3 & 8,5 & $\begin{array}{l}25,6 \\
77,7\end{array}$ & $\begin{array}{r}4,0 \\
11,0\end{array}$ & $\begin{array}{l}0,151 \\
0,1+3\end{array}$ & $\begin{array}{l}0,98 \\
0,99\end{array}$ \\
\hline 1,8 & 58,3 & 24,4 & $\begin{array}{l}20,5 \\
44,5\end{array}$ & $\begin{array}{l}14,4 \\
30,8\end{array}$ & $\begin{array}{l}0,70 \\
0,69\end{array}$ & $\begin{array}{l}0,615 \\
0,61\end{array}$ \\
\hline 1,4 & 175 & 8,5 & $\begin{array}{l}33,0 \\
31,5 \\
55,1\end{array}$ & $\begin{array}{l}2,55 \\
2,7 \\
4,65\end{array}$ & $\begin{array}{l}0,077 \\
0,086 \\
0,084\end{array}$ & $\begin{array}{l}0,91 \\
0,94 \\
0,98\end{array}$ \\
\hline 1,5 & 175 & 8,5 & $\begin{array}{l}20,1 \\
36,7 \\
49,0\end{array}$ & $\begin{array}{l}2,3 \\
3,9 \\
5,0\end{array}$ & $\begin{array}{l}0,114 \\
0,106 \\
0,102\end{array}$ & $\begin{array}{l}0,96 \\
1,00 \\
1,00\end{array}$ \\
\hline 1,6 & 175 & 8,5 & $\begin{array}{l}31,0 \\
31,5 \\
53,3\end{array}$ & $\begin{array}{l}3,2 \\
3,45 \\
5,2\end{array}$ & $\begin{array}{l}0,103 \\
0,109 \\
0,98\end{array}$ & $\begin{array}{l}1,00 \\
0,935 \\
1,00\end{array}$ \\
\hline 1,78 & 175 & 8,5 & $\begin{array}{l}15 \\
30 \\
44\end{array}$ & $\begin{array}{l}1,6 \\
3,5 \\
5,45\end{array}$ & $\begin{array}{l}0,106 \\
0,117 \\
0,127\end{array}$ & $\begin{array}{l}0,97 \\
0,915 \\
0,99\end{array}$ \\
\hline 1,0 & 175 & 15 & $\begin{array}{l}35,6 \\
49,4\end{array}$ & $\begin{array}{l}2.2 \\
3.3\end{array}$ & $\begin{array}{l}0,062 \\
0,067\end{array}$ & $\begin{array}{l}0,91 \\
0,995\end{array}$ \\
\hline 1,4 & 175 & 15 & $\begin{array}{l}17,9 \\
36,9 \\
37,8\end{array}$ & $\begin{array}{l}2,4 \\
4,9 \\
5,15\end{array}$ & $\begin{array}{l}0,135 \\
0,133 \\
0,135\end{array}$ & $\begin{array}{l}0,98 \\
0,97 \\
0,935\end{array}$ \\
\hline 1,8 & 175 & 15 & $\begin{array}{l}18,5 \\
35,4 \\
65,0\end{array}$ & $\begin{array}{r}3,3 \\
7,5 \\
13,2\end{array}$ & $\begin{array}{l}0,178 \\
0,21 \overline{2} \\
0,203\end{array}$ & $\begin{array}{l}0,80 \\
1,00 \\
1,00\end{array}$ \\
\hline 0,8 & 175 & 27,5 & $\begin{array}{l}24,8 \\
30,7\end{array}$ & $\begin{array}{l}2,7 \\
+1,1\end{array}$ & $\begin{array}{l}0,108 \\
0,133\end{array}$ & $\begin{array}{l}0,91 \\
0,88\end{array}$ \\
\hline
\end{tabular}

V. - Variation de la résistance

Afin de vérifier si les formules théoriques qui tiennent conpte d'une résistance au mouvement ont une signification, nous arons introduit une résistance additionnelle variable. Pour obtenir cette résistance, on a placé au-dessous de l'obstacle un filtre constitué soit par une plaque perforée, soit par une caisse en métal déployé remplie de paille d'aluminium.

Sur la figure 24, nous avous représenté les valeurs théoriques et les résultats expérimenlaux avec un systime de coordonnées $r, t$ et $p, t$. La courbe théorique $r, t$, a un minimum $r=0,55$, tandis que le minimum expérimental est de $\mathbf{0 , 5 8 5}$.

Les valeurs expérimentales pour p suivent assez bien la courbe théorique, et présentent comme celle-ci un maximum. 
Fig. 20

Rapport des amplitudes : $t=\left(2 a_{2} / 2 a_{1}\right)$

$$
t_{0}=\frac{1}{\sqrt{1+1-\eta^{2}}} \quad \eta=\frac{2 x^{2} l l}{g \mathrm{~T}-2}
$$
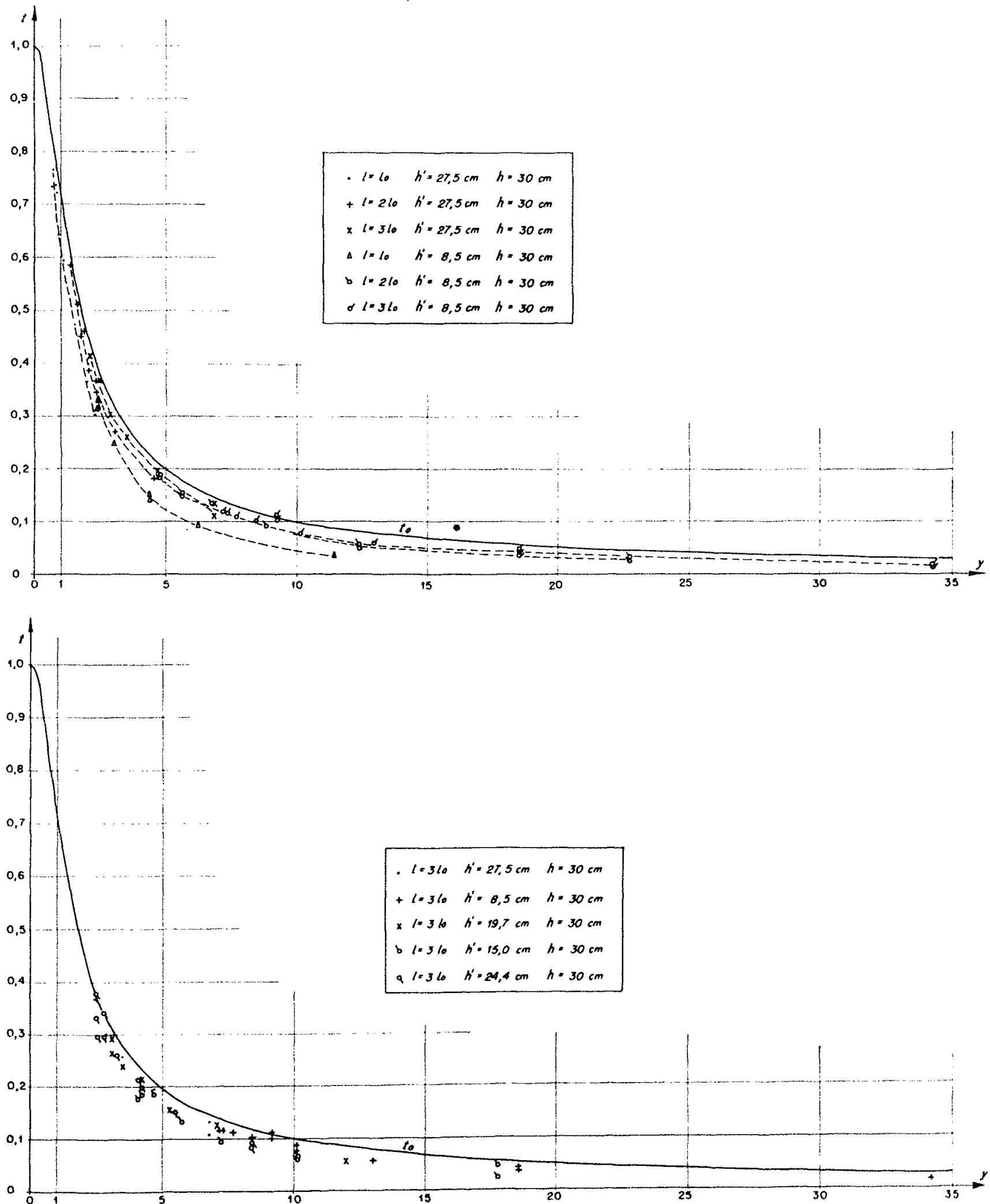
Fig. 21

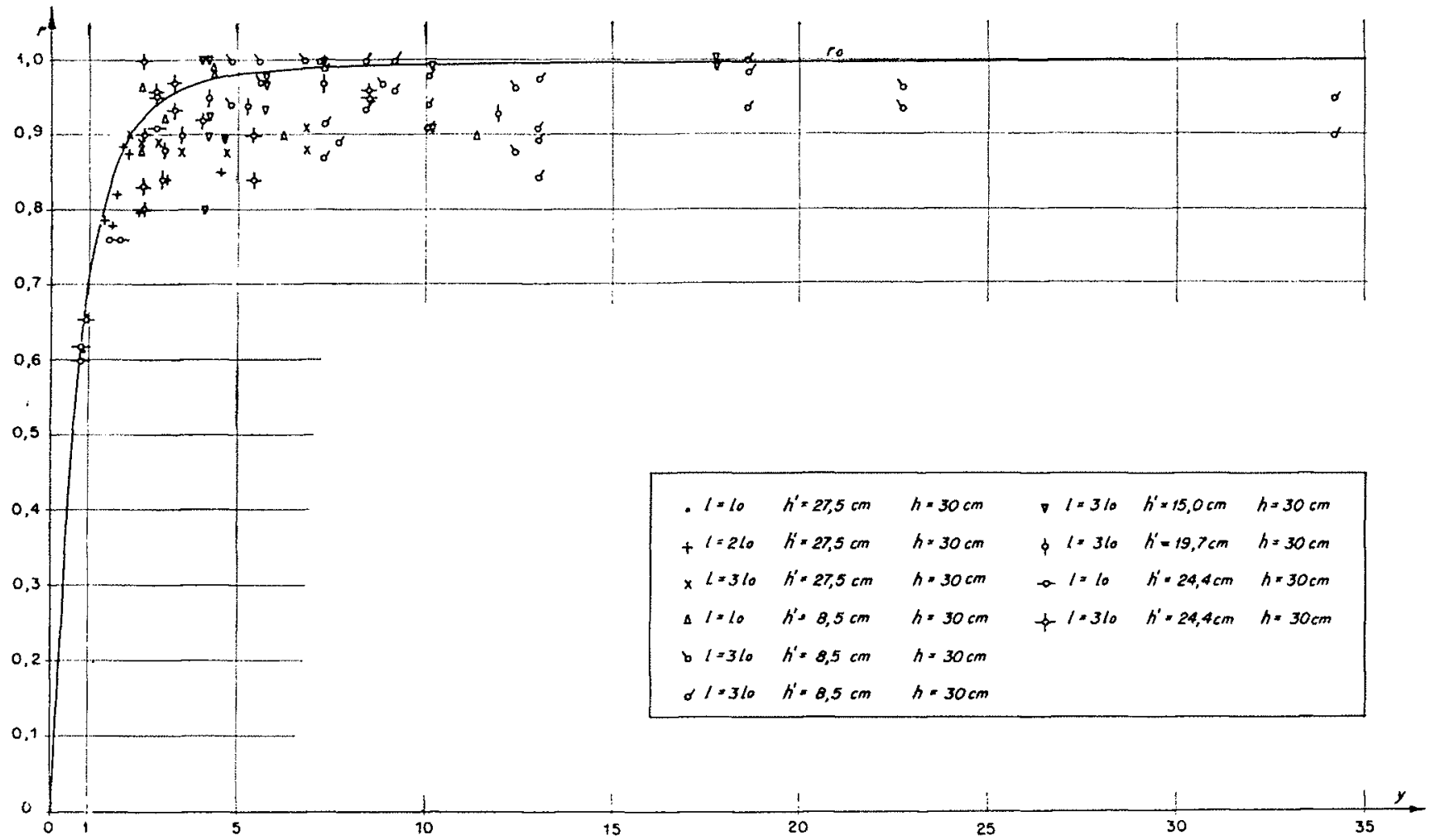

Rapport des amplitudes : $r=\left(2 a_{3} / 2 a_{1}\right)$

$$
r_{0}=\frac{y}{\sqrt{1+y^{2}}} \quad y=\frac{2 \pi^{2} l f}{g \mathrm{~T}^{2}}
$$

Fig. 22
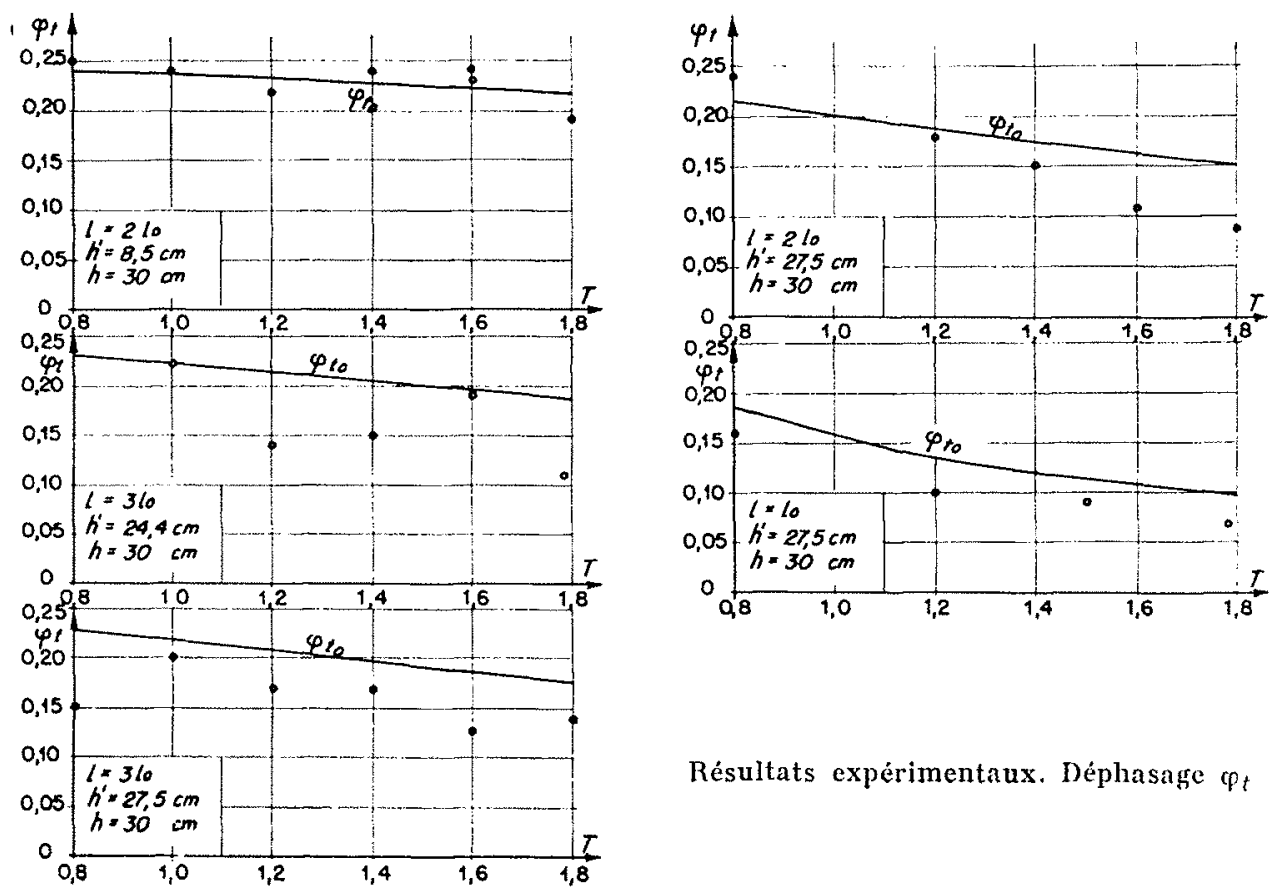

Résultats expérimentaux. Déphasage $\varphi_{t}$ 


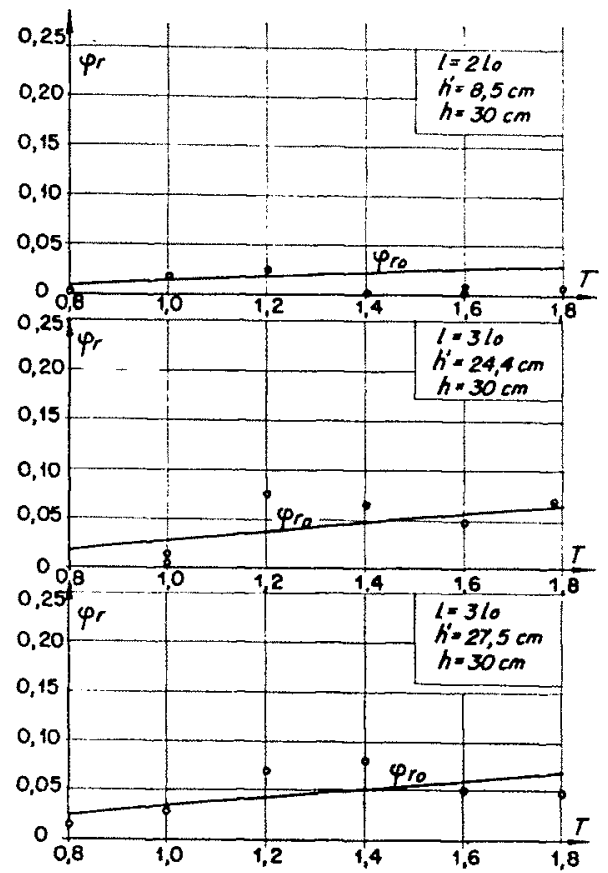

Au cours des expériences, nous avons constaté le déplacement de nouds et de ventres. Au fur et à mesure qu'on augsmentait la résistance, ils se déplaçaient vers l'obstacle.

On a représenté les résultats d'une autre facon sur la figure 25 . On y a construit des triangles, avec les segments $I, T, R$ qui ont comme modules, respectivement, l'unité $r$ et $t$. La théorie établit que $A_{1}=A_{2}+A_{3}$, done que $\vec{T}=\vec{R}+\vec{T}$. l'angle entre $I$ et $R$ donne le déphasage entre la houle transmise et la houle incidente, tandis que l'angle entre $\mathbf{I}$ et $\mathrm{R}$ donne le déphasage entre la houle réfléchie et la houle incidente. On y voit bien que les deıx déphasages diminuent au fur et à mesure que la résistance augmente.

\section{VI. - Comparaison avec les expériences} du passage de la houle sous une vanne plate

M. SkLÉnard donne une série des courbes qui représentent ses résultats expérimentaux pour différentes hauteurs d'eau et pour les périodes de $0,96 \mathrm{~s}$ et $1,45 \mathrm{~s}$. Sur les courbes correspondant à $h=0,30 \mathrm{~m}$, nous avons pris les valeurs moyennes du rapport $t$ que l'on reproduit dans le tableau suivant. De nos graphiques des résultats expérimentaux, on a déduit les valeurs correspondantes, pour une longueur de l'obstacle $l=58,3 \mathrm{~cm}$, afin de les comparer avec celles de M. SKLÉNARD.

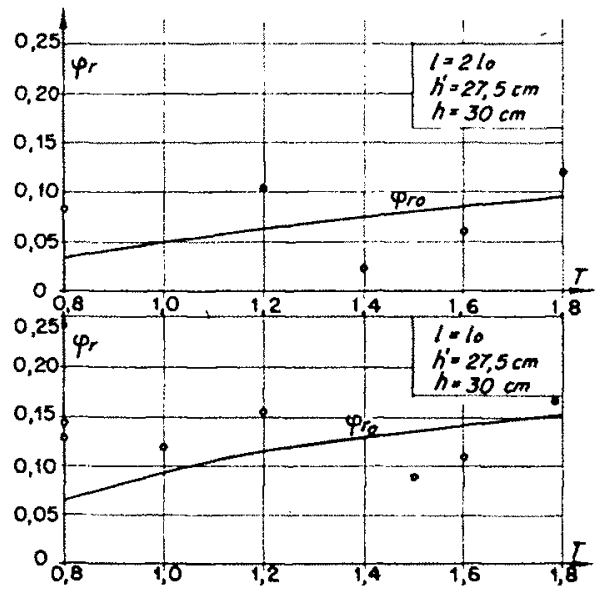

FIG. 23.

Résultats expérimentaux. Déphasage $\varphi_{1}$.

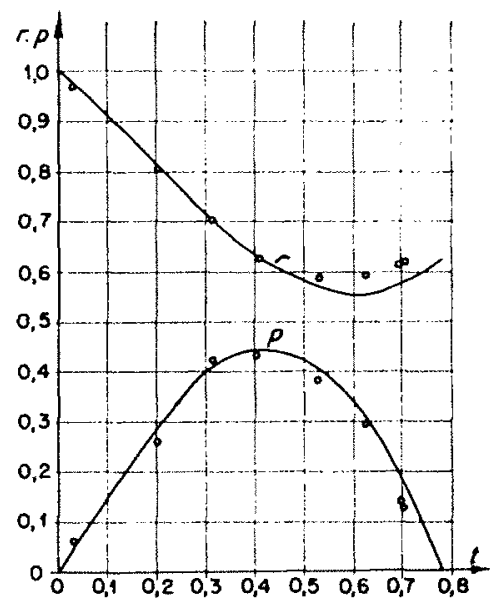

Fili. 24.

Essai avec variation de la résistance.

$$
\begin{array}{ll}
l=58,3 \mathrm{~cm} \quad h=30 \mathrm{~cm} & h^{\prime}=24,4 \mathrm{~cm} \\
r=\frac{1}{\sqrt{(1+x)^{2}+y^{2}}} & x=\frac{\pi c /}{\mathrm{T}} \\
t=\sqrt{\frac{x^{2}+y^{2}}{(1+x)^{2}+y^{2}}} & y=\frac{2 \pi^{2} l f}{g \mathrm{~T}^{2}} \\
p=\frac{2 x}{(1+x)^{2}+y^{2}} & f=\frac{\operatorname{ch~} m h}{\operatorname{ch} m h^{\prime}}
\end{array}
$$




\begin{tabular}{|c|c|c|c|c|c|}
\hline$h^{\prime} / h$ & 0,283 & 0,815 & 0,92 & 1 & $\mathrm{~T}$ \\
\hline $1=0$ & 0.72 .7 & 0.987 & 0,9915 & 1.00 & \multirow{2}{*}{$1,45 \mathrm{~s}$} \\
\hline$l=58,3 \mathrm{~cm}$ & 0,23 & 0,58 & 0,68 & 0,75 & \\
\hline$l=0$ & 0,31 & 0,953 & 1 & 1 & \multirow{2}{*}{$0,96 \mathrm{~s}$} \\
\hline$l=58,3 \mathrm{~cm}$ & $0,0 \mathrm{~s}$ & 0,345 & 0,44 & 0,48 & \\
\hline
\end{tabular}

Pour faire la comparaison plus complète, on a réalisé quelques mesures pour $h^{\prime}=30 \mathrm{~cm}$ et $l=58,3 \mathrm{~cm}$ (fig. 26), les essais ont montré que, dans ces conditions, Ie rapport $t$ reste bien inféricur à l'unité. La mesure dans ce cas est, cependant, imprécise; cette imprécision étant due à l'entrée d'air qui se produit en-dessous de l'obstacle, aux deux extrémités, quand le niveau descend. Quand il monte, l'air s'echappe en produisant une perturbation du mouvement. Pour des grandes amplitudes, il se produit, en plus, un passage de grosses bulles d'air de l'amont vers l'aval, ce qui rend encore plus irrégulier le phénomène. De toute façon, on n'est pas arrivé à avoir une surface libre continue entre l'amont et l'aval, le passage restant toujours en charge.

\section{VII. - Conclusions}

L'étude expérimentale de la houle dans un cinal présentant un passage en charge, que nous venons d'exposer ici, correspond à une zone très réduite du domaine de variation des divers paramétres du phénomene. L'extrapolation par les lois de la similitude dynamique permettrait, pourtant, de l'agrandir, mais nous préférons ne pas arancer une affirmation dans ce sens-là.

précisons done bien les limites entre lesquelles nous avons fait les expériences.

$$
\begin{aligned}
& 0,8 \mathrm{~s} \leqslant \mathrm{~T} \leqslant 1,8 \mathrm{~s} \\
& 58,3 \mathrm{~cm} \leqslant l \leqslant 175 \quad(\mathrm{~m} \\
& 20 \mathrm{~cm} \leqslant h \leqslant 30 \mathrm{~cm} \\
& 8,5 \mathrm{~cm} \leqslant h^{\prime} \leqslant 30 \mathrm{~cm} \\
& 0,8 \mathrm{~cm} \leqslant 2 a_{1} \leqslant 7,7 \mathrm{~cm}
\end{aligned}
$$

Entre ces limites, une théorie élémentaire nous donne des formules pour les rapports $t$ et $r$ qui limitent supérieurement les valeurs expérimenlales. Une limite inférieure est donne approxi-

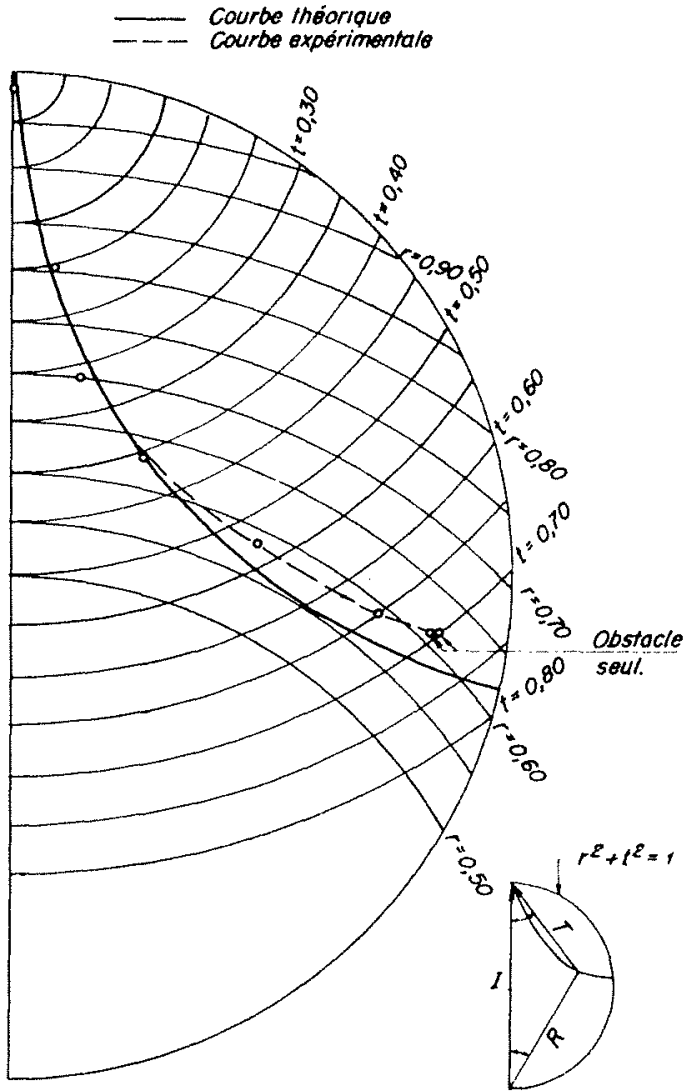

Fig. 25. Essai avec variation de la rèsistance. $l=58,3 \mathrm{~cm} ; \quad h=30 \mathrm{~cm} ; \quad h^{\prime}=24,4 \mathrm{~cm}$
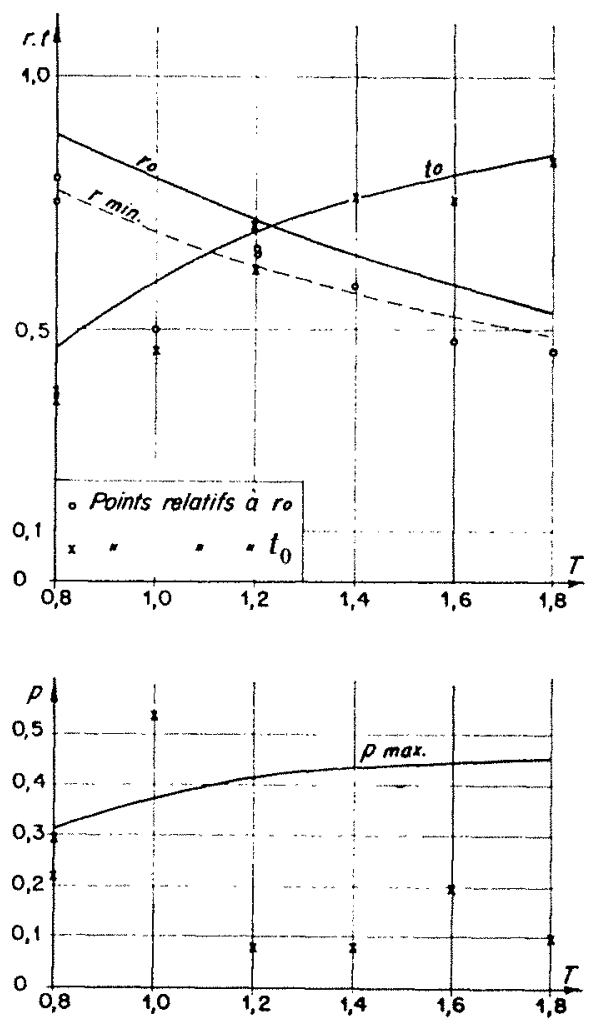

Fig. 26. Résultats expérimentaux $(r, t, p)$. $l=58,3 \mathrm{~cm} ; \quad h=30 \mathrm{~cm} ; \quad h^{\prime}=30 \mathrm{~cm}$ 
mativement par la formule de $r_{\text {min. }}$. De mème une limite supérieure pour la perte relative d'énergie est donnée par la formule de $p_{\max }$.

Laccord entre théorie et expérience est meilleur pour les grandes valeurs de T, $l, h^{\prime}$.
1) point de vue cmpirique, on peut fixer asse\% bien les valeurs du rapport $t$, mais un calcul avec un coefficient de résistance fixè à priori nous semble aléatoire. Pour $r, p$ et les déphasages $\vartheta_{t}$ et $\varphi_{r}$ on peut fixer des limites approximalives.

\section{ANNEXE I}

COMPARAISONS ENTRE VALEURS THÉORIQUES ET EXPERIMENTALES

TABLEAU 1

\begin{tabular}{|c|c|c|c|c|c|c|c|}
\hline \multicolumn{3}{|c|}{$l=175 \mathrm{~cm}$} & \multicolumn{7}{c|}{$h=30 \mathrm{~cm}$} & $h^{\prime}=27,5 \mathrm{~cm}$ \\
\hline $\mathrm{T} s$ & $t$ & $t_{0}$ & $r_{m i n}$ & $r$ & $r_{0}$ & $p$ & $p_{\max }$ \\
\hline & & & & & & & \\
\hline 0,8 & 0,133 & 0,146 & 0,93 & 0,88 & 0,989 & 0,208 & 0,127 \\
0,8 & 0,108 & 0,146 & 0,93 & 0,91 & 0,989 & 0,159 & 0,127 \\
1 & 0,195 & 0,219 & 0,90 & 0,875 & 0,976 & 0,196 & 0,180 \\
1,2 & 0,256 & 0,276 & 0,87 & 0,88 & 0,961 & 0,162 & 0,216 \\
1,4 & 0,300 & 0,332 & 0,84 & 0,89 & 0,943 & 0,116 & 0,249 \\
1,6 & 0,367 & 0,384 & 0,815 & 0,89 & 0,923 & 0,074 & 0,277 \\
1,8 & 0,412 & 0,431 & 0,785 & 0,90 & 0,902 & 0,020 & 0,301 \\
& & & & & & & \\
\hline
\end{tabular}

TABLEAU 2

\begin{tabular}{|c|c|c|c|c|c|c|c|}
\hline \multicolumn{3}{|c|}{$l=175 \mathrm{~cm}$} & \multicolumn{3}{|c|}{$h=30 \mathrm{~cm}$} & \multicolumn{2}{|c|}{$h^{\prime}=24,4 \mathrm{~cm}$} \\
\hline $\mathrm{T} s$ & $t^{1}$ & $t_{0}$ & $r_{\min }$ & $r$ & $r_{0}$ & $p$ & $p_{m a x}$ \\
\hline 0,73 & 0,061 & 0,096 & 0,95 & 0,845 & 0,997 & 0,282 & 0,087 \\
\hline 0,73 & 0,069 & 0,096 & 0,95 & 0,885 & 0,997 & 0,212 & 0,087 \\
\hline 0,8 & 0,093 & 0,118 & 0,94 & 0,95 & 0,993 & 0,089 & 0,105 \\
\hline 0,8 & 0,086 & 0,118 & 0.94 & 0,96 & 0,993 & 0,071 & 0,105 \\
\hline 1,0 & 0,152 & 0,181 & 0,92 & 0,90 & 0,983 & 0,167 & 0,154 \\
\hline 1,0 & 0,148 & 0,181 & 0,92 & 0,84 & 0,983 & 0,272 & 0,154 \\
\hline 1,2 & 0,218 & 0,239 & 0,885 & 0,92 & 0,971 & 0,160 & 0.193 \\
\hline 1,4 & 01,264 & 0.292 & 0.86 & 0.97 & 0.956 & -0.015 & 0,226 \\
\hline 1,4 & 0,260 & 0,292 & 0,86 & 0,935 & 0,956 & 0,058 & 0,226 \\
\hline 1,6 & 0,340 & 0,341 & 0,83 & 0,96 & 0,940 & $-0,048$ & 0,255 \\
\hline 1,6 & 0,290 & 0,341 & 0,83 & 0,95 & 0,940 & 0,012 & 0,255 \\
\hline 1,78 & 0,333 & 0,382 & 0,81 & 1,00 & 0,920 & $-0,110$ & 0,277 \\
\hline 1,78 & 0,380 & 0,382 & 0,81 & 0,90 & 0,920 & 0,043 & 0,277 \\
\hline 1,78 & 0,333 & 0,382 & 0,81 & 0,83 & 0,920 & 0,200 & 0,277 \\
\hline 1,78 & 0,295 & 0,382 & 0,81 & 0,80 & 0,920 & 0,273 & 0.277 \\
\hline
\end{tabular}

TABLEAU 3

\begin{tabular}{|c|c|c|c|c|c|c|c|}
\hline \multicolumn{3}{|c|}{$l=175 \mathrm{~cm}$} & \multicolumn{3}{|c|}{$h=30 \mathrm{~cm}$} & \multicolumn{2}{|c|}{$h=19.7 \mathrm{~cm}$} \\
\hline $\mathrm{T}$ s & $t$ & $t_{0}$ & $r_{m i n}$ & $r$ & $r_{0}$ & $p$ & $p_{m a t}$ \\
\hline 0,8 & 0,055 & 0,0835 & 0,96 & 0,93 & 0,996 & 0,132 & 0,077 \\
\hline 1,0 & 0,122 & 0,111 & 0,93 & 0.97 & 0,990 & 0,044 & 0,124 \\
\hline 1,2 & 0,155 & 0,187 & 0,905 & 0,94 & 0,982 & 0,092 & 0,158 \\
\hline 1,4 & 0,208 & 0,233 & 0,89 & 0,95 & 0,972 & $0,05.4$ & 0,189 \\
\hline 1,6 & 0,238 & 0,276 & 0,87 & 0,90 & 0,961 & 0,134 & 0,217 \\
\hline 1,8 & 0,260 & 0,315 & 0,84 & 0,84 & 0,948 & 0,227 & 0,241 \\
\hline 1,8 & 0,292 & 0,315 & 0,84 & 0.88 & 0,948 & 0,140 & 0,241 \\
\hline
\end{tabular}

TABLEAU 4

\begin{tabular}{|c|c|c|c|c|c|c|c|}
\hline \multicolumn{3}{|c|}{$l=175 \mathrm{~cm}$} & \multicolumn{3}{|c|}{$h=30 \mathrm{~cm}$} & \multicolumn{2}{|c|}{$h^{\prime}=15 \mathrm{~cm}$} \\
\hline $\mathrm{T}$ s & $t$ & $t_{0}$ & $r_{m i n}$ & $r$ & $r_{0}$ & $p$ & $p_{\max }$ \\
\hline 0,8 & 0,021 & $0,0,86$ & 0,975 & 0,99 & 0,998 & 0.102 & 0,053 \\
\hline 0,9 & 0,0465 & 0.077 & 0,966 & 1,00 & 0,997 & $-0,002$ & 0,072 \\
\hline 1,0 & 0,0667 & 0.0998 & 0,956 & $0,99 \%$ & 0,995 & 0,006 & 0,089 \\
\hline 1,0 & 0.062 & 0.098 & 0,956 & 0,91 & 0,995 & 0,17 & 0,089 \\
\hline 1,2 & 0,095 & 0.137 & 0,935 & 1,00 & 0,99 & $-0,009$ & 0,120 \\
\hline 1,4 & 0.134 & 0,173 & 0,92 & 0,98 & 0,985 & 0,02 & 0,148 \\
\hline 1,4 & 0,133 & 0,173 & 0,92 & 0,97 & 0,985 & 0,04 & 0,148 \\
\hline 1,4 & 0,136 & 0,173 & 0,92 & 0.935 & 0,985 & 0,11 & 0,148 \\
\hline 1.61 & 0,185 & 0,214 & 0,90 & 0,895 & 0,978 & 0,165 & 0,174 \\
\hline 1.78 & 0,185 & 0,235 & 0,88 & 0,93 & 0,972 & 0,161 & 0,191 \\
\hline 1,78 & 0,189 & 0,235 & 0,88 & 0,90 & 0,972 & 0,155 & 0.191 \\
\hline 1,8 & 0,202 & 0.239 & 0,875 & 0,90 & 0,97 & 0,15 & 0,193 \\
\hline 1,8 & 0.178 & 0,239 & 0,875 & 0,90 & 0,97 & 0,16 & 0,193 \\
\hline
\end{tabular}


TABLLAU :

\begin{tabular}{|c|c|c|c|c|c|c|c|}
\hline \multicolumn{3}{|c|}{$l=175 \mathrm{~cm}$} & \multicolumn{3}{|c|}{$h=30 \mathrm{~cm}$} & \multicolumn{2}{|c|}{$h^{\prime}=8,5 \mathrm{~cm}$} \\
\hline $\mathrm{Ts}$ & $t$ & $t_{0}$ & $r_{m i n}$ & $r$ & $r_{0}$ & $p$ & $p_{\max }$ \\
\hline 0,8 & 0,0164 & 0,0295 & 0,98 & 0,95 & 0,999 & 0,097 & 0,0287 \\
\hline 0,8 & 0,0136 & 0,0295 & 0,98 & 0,90 & 0,999 & 0,190 & 0,0287 \\
\hline 1,0 & 0,0406 & 0,0540 & 0,97 & 1,00 & 0,998 & $-0,002$ & 0,0512 \\
\hline 1,0 & 0,0362 & 0,0540 & 0,97 & 0,985 & 0,998 & 0,029 & 0,0512 \\
\hline 1,0 & 0,0403 & 0,0540 & 0,97 & 0,935 & 0,998 & 0,124 & 0,0512 \\
\hline 1,2 & 0,0575 & 0,0775 & 0,96 & 0,91 & 0,997 & 0,168 & 0,0719 \\
\hline 1,2 & $0,0560\}$ & 0,0775 & 0,96 & 0,895 & 0,997 & 0,196 & 0,0719 \\
\hline 1.2 & 0,0580 & 0,0775 & 0,96 & 0,975 & 0,997 & 0,647 & 0,0719 \\
\hline 1,4 & 0,0775 & 0,099 & 0,95 & 0,91 & 0,995 & 0,166 & 0,090 \\
\hline 1,4 & 0,0860 & 0,099 & 0,95 & 0,94 & 0,995 & 0,108 & 0,090 \\
\hline 1,4 & 0,0845 & 0,099 & 0,95 & 0,98 & 0,995 & 0,033 & 0,090 \\
\hline 1.5 & 0,114 & 0,109 & 0,945 & 0,96 & 0,994 & 0,078 & 0,098 \\
\hline 1,5 & 0,106 & 0,109 & 0,945 & 1,00 & 0,994 & $-0,011$ & 0,098 \\
\hline 1,5 & 0,102 & 0,109 & 0,945 & 1,00 & 0,994 & $-0,010$ & 0,098 \\
\hline 1,6 & 0,098 & 0,119 & 0,94 & 1,00 & 0,993 & $-0,009$ & 0,106 \\
\hline 1,6 & 0,103 & 0,119 & 0,94 & 1,00 & 0,993 & $-0,011$ & 0,106 \\
\hline 1,6 & 0.109 & 0,119 & 0,94 & 0,935 & 0,993 & 0,114 & 0,106 \\
\hline 1,7 & 0,110 & 0,129 & 0,935 & 0,89 & 0.9915 & 0.196 & 0,114 \\
\hline 1,78 & 0,116 & 0,137 & 0,93 & 0.915 & 0,990 & 0,149 & 0,120 \\
\hline 1,78 & 0,105 & 0,137 & 0,93 & 0,87 & 0,990 & 0,232 & 0,120 \\
\hline 1,78 & 0,126 & 0,137 & 0,93 & 0,99 & 0,990 & 0,004 & 0,120 \\
\hline 1,8 & 0,116 & 0,138 & 0,93 & 1,00 & 0,990 & $-0,013$ & 0,122 \\
\hline
\end{tabular}

TABLEAU 6

\begin{tabular}{|c|c|c|c|c|c|c|c|}
\hline \multicolumn{3}{|c|}{$l=116,7 \mathrm{~cm}$} & \multicolumn{3}{c|}{$h=30 \mathrm{~cm}$} & $h^{\prime}=27,5 \mathrm{~cm}$ \\
\hline $\mathrm{T} s$ & $t$ & $t_{0}$ & $r_{\text {min }}$ & $r$ & $r_{0}$ & $p$ & $p_{\text {ma } .}$ \\
\hline & & & & & & & \\
\hline 0,8 & 0,181 & 0,216 & 0,895 & 0,85 & 0,975 & 0,245 & 0,178 \\
1 & 0,270 & 0,312 & 0,85 & 0,84 & 0,95 & 0,221 & 0,238 \\
1,2 & 0,344 & 0,395 & 0,81 & 0,80 & 0,92 & 0,242 & 0,293 \\
1,2 & 0,367 & 0,395 & 0,81 & 0,80 & 0,92 & 0,232 & 0,293 \\
1.3 & 0,384 & 0,431 & 0,79 & 0,875 & 0,90 & 0,093 & 0,301 \\
1,4 & 0,461 & 0,467 & 0,775 & 0,885 & 0,885 & 0,005 & 0,318 \\
1,5 & 0,450 & 0,498 & 0,76 & 0,82 & 0,865 & 0,123 & 0,332 \\
1,6 & 0,510 & 0,530 & 0,750 & 0,78 & 0,85 & 0,139 & 0,346 \\
1,8 & 0,586 & 0,582 & 0,705 & 0,785 & 0,815 & 0,041 & 0,368 \\
\hline
\end{tabular}

TABLEAU 7

\begin{tabular}{|c|c|c|c|c|c|c|c|}
\hline \multicolumn{3}{|c|}{$l=-116,7 \mathrm{~cm}$} & \multicolumn{3}{|c|}{$h=30 \mathrm{~cm}$} & \multicolumn{2}{|c|}{$h^{\prime}=24.4 \mathrm{~cm}$} \\
\hline $\mathbf{T} \mathrm{s}$ & $t$ & $t_{0}$ & $r_{m i n}$ & $r$ & $r_{0}$ & $p$ & $p_{m a x}$ \\
\hline 0,8 & 0,120 & 0,175 & 0,915 & 0,90 & 0,985 & 0,420 & 0,149 \\
\hline 1 & 0,224 & 0,267 & 0,885 & 0,89 & 0,965 & 0,400 & 0,211 \\
\hline 1,3 & 0,337 & 0,382 & 0,82 & 0,90 & 0,925 & 0,370 & 0,276 \\
\hline 1,6 & 0,392 & 0,479 & 0,77 & 0,84 & 0,87 & 0,254 & 0,324 \\
\hline 1,8 & 0,479 & 0,531 & 0,735 & 0,87 & 0,845 & 0,187 & 0,347 \\
\hline 1.8 & 0,494 & 0,531 & 0,735 & 0,89 & 0,845 & $-0,155$ & 0,347 \\
\hline \multicolumn{8}{|c|}{ TABLEAU 8} \\
\hline \multicolumn{3}{|c|}{$l=116,7 \mathrm{~cm}$} & \multicolumn{3}{|c|}{$h=30 \mathrm{~cm}$} & \multicolumn{2}{|c|}{$h^{\prime}=8,5 \mathrm{~cm}$} \\
\hline $\mathrm{Ts}$ & $t$ & $t_{0}$ & $r_{\min }$ & $r$ & $r_{0}$ & $p$ & $p_{\max }$ \\
\hline 0,8 & 0,0236 & 0,044 & 0,975 & 0,965 & 0,999 & 0,068 & 0,042 \\
\hline 0,8 & 0,0302 & 0,044 & 0,975 & 0,935 & 0,999 & 0,125 & 0,042 \\
\hline 1,0 & 0,056 & 0,081 & 0,96 & 0,905 & 0,997 & 0,065 & 0,075 \\
\hline 1,2 & 0,093 & 0,115 & 0,945 & 0,97 & 0,993 & 0,051 & 0,103 \\
\hline 1,4 & 0,131 & $0,1+7$ & 0,93 & 1,00 & 0,989 & $-0,018$ & 0,128 \\
\hline 1,4 & 0,136 & 0.147 & 0,93 & 1,00 & 0,989 & $-0,018$ & 0,128 \\
\hline 1,6 & 0,149 & 0,177 & 0,92 & 0,97 & 0,984 & 0,037 & 0,151 \\
\hline 1.6 & 0,153 & 0,177 & 0,92 & 1,00 & 0,984 & $\ldots 0,023$ & 0.151 \\
\hline 1,8 & 0,189 & $0,20 \vdots$ & 0,905 & 1,00 & 0,974 & $-0,036$ & 0,170 \\
\hline 1,8 & 0,181 & 0,205 & 0,905 & 0,94 & 0,974 & 0,084 & 0,170 \\
\hline
\end{tabular}

TABLEAU 9

\begin{tabular}{|c|c|c|c|c|c|c|c|}
\hline \multicolumn{3}{|c|}{$l=58,3 \mathrm{~cm}$} & \multicolumn{3}{|c|}{$h=30 \mathrm{~cm}$} & \multicolumn{2}{|c|}{$h^{\prime}=27,5 \mathrm{~cm}$} \\
\hline $\mathrm{Ts}$ & $t$ & $t_{0}$ & $r_{m i n}$ & $r$ & $r_{0}$ & $p$ & $p_{m a, x}$ \\
\hline 0,8 & 0,307 & 0,405 & 0,805 & 0,80 & 0,85 & 0,266 & 0.288 \\
\hline 0,8 & 0,301 & 0,405 & 0,805 & 0,80 & 0,85 & 0,269 & 0,288 \\
\hline 1 & 0,473 & 0,549 & 0,725 & 0,76 & 0,835 & 0,200 & 0,355 \\
\hline 1,2 & 0,594 & 0,654 & 0,66 & 0,665 & 0,755 & 0,205 & 0,395 \\
\hline 1,4 & 0,641 & 0,730 & 0,60 & 0,66 & 0,685 & 0,153 & 0,422 \\
\hline 1,5 & 0,72 & 0,755 & 0,58 & 0,61 & 0,655 & 0,110 & 0,430 \\
\hline 1,5 & 0,72 & 0,755 & 0,58 & 0,57 & 0,655 & 0,156 & 0,430 \\
\hline 1,6 & 0,735 & 0,781 & 0,555 & 0,615 & 0,625 & 0,080 & 0,439 \\
\hline 1,78 & 0,765 & 0,817 & 0.510 & 0,49 & 0,575 & $0,17 \vdots$ & 0,449 \\
\hline 1,8 & 0,735 & 0,820 & 0,515 & 0,445 & 0,57 & 0,261 & 0,450 \\
\hline
\end{tabular}


TABLEAL 10

\begin{tabular}{|c|c|c|c|c|c|c|c|}
\hline \multicolumn{3}{|c|}{$l=58,3 \mathrm{~cm}$} & \multicolumn{3}{c|}{$\mathrm{h}=30 \mathrm{~cm}$} & $h^{\prime}=24,4 \mathrm{~cm}$ \\
\hline $\mathrm{T} \mathrm{s}$ & $t$ & $t_{0}$ & $r_{\min }$ & $r$ & $r_{0}$ & $p$ & $p_{m n . .}$ \\
\hline & & & & & & & \\
\hline 0,8 & 0,223 & 0,335 & 0,84 & 0,91 & 0,94 & 0,12 & 0,251 \\
1,0 & 0,372 & 0,484 & 0,765 & 0,76 & 0,875 & 0,28 & 0,326 \\
1,3 & 0,476 & 0,635 & 0,67 & 0,74 & 0,77 & 0,23 & 0,388 \\
1,6 & 0,649 & 0,737 & 0,595 & 0,655 & 0,675 & 0,15 & 0,424 \\
1,8 & 0,695 & 0,782 & 0,550 & 0,61 & 0,625 & 0,16 & 0,439 \\
1,8 & 0,70 & 0,782 & 0,550 & 0,615 & 0,625 & 0,13 & 0,439 \\
\hline
\end{tabular}

TABLEAU 11

\begin{tabular}{|c|c|c|c|c|c|c|c|}
\hline \multicolumn{3}{|c|}{$l=58,3 \mathrm{~cm}$} & \multicolumn{3}{|c|}{$h=30 \mathrm{~cm}$} & \multicolumn{2}{|c|}{$h^{\prime}=8,5 \mathrm{~cm}$} \\
\hline $\mathrm{Ts}$ & $t$ & $i_{0}$ & $r_{m i n}$ & $r$ & $r_{0}$ & $p$ & $p_{m a t}$ \\
\hline 0,8 & 0,0345 & 0.087 & 0,95 & 0,90 & 0,995 & 0,18 & 0,080 \\
\hline 1 & 0,0935 & 0,160 & 0,925 & 0,90 & 0,987 & 0,19 & 0,138 \\
\hline 1,2 & 0,151 & 0,226 & 0,89 & 0,98 & 0,974 & 0,017 & 0,184 \\
\hline 1,2 & 0.144 & 0,226 & 0,89 & 0,99 & 0,974 & $-0,0007$ & 0,184 \\
\hline 1,5 & 0,243 & 0,312 & 0,85 & 0,92 & 0,950 & 0,095 & 0,237 \\
\hline 1,8 & 0,33 & 0,386 & 0.81 & 0,98 & 2 & 0,114 & 0,279 \\
\hline 1,8 & 0,315 & 0,386 & 0,81 & 0,965 & 0,922 & $-0,029$ & 0,279 \\
\hline
\end{tabular}

\section{A SUGGESTION...}

You have obtained this number because one of the articles in it coincides with your present activities. The other articles and numbers seem to be of no practical use to you.

But do you know how you will be preoccupied in a few years, or even in a few months? The number which you may now consider as uninteresting can then become an absolutely indispensable adjunct to your work. You will ask us for it and, as occurs every day, we shall reply that it is out of print.

TAKE OUT A SUBSCRIPTION; glance through each number when you receive it. The articles may perhaps be of no help to you today, but tomorrow you will know that they exist and, when the need arises, you will find them with no difficulty in YOUR own collection.

TAKE OUT A SUBSCRIPTION; it is safer, easier and much cheaper.

\section{LA HOUILLE BLANCHE.}

